\title{
Damage Detection of Shear Connectors in Bridge Structures with Transmissibility in Frequency Domain
}

\author{
Jun $\mathrm{Li}^{1{ }^{1, *}}$, Hong $\mathrm{Hao}^{1}$, Yong $\mathrm{Xia}^{2}$ and Hong-ping $\mathrm{Zhu}^{3}$ \\ ${ }^{1}$ School of Civil and Resource Engineering, The University of Western Australia, \\ 35 Stirling Highway, Crawley, WA 6009, Australia \\ Email: jun.li@uwa.edu.au and hong.hao@uwa.edu.au. \\ ${ }^{2}$ Department of Civil and Environmental Engineering, The Hong Kong Polytechnic University \\ Hung Hom, Kowloon, Hong Kong. Email: ceyxia@polyu.edu.hk. \\ ${ }^{3}$ School of Civil Engineering and Mechanics, Huazhong University of Science and Technology \\ Wuhan, Hubei, People’s Republic of China. Email: hpzhu@mail.hust.edu.cn
}

Received 7 February 2013

Accepted 27 August 2013

\begin{abstract}
Shear connectors are generally used to link the slab and girder together in slab-on-girder bridge structures. Damage of shear connectors in such structures will result in shear slippage between the slab and girder, which significantly reduces the load-carrying capacity of bridges. A damage detection approach based on transmissibility in frequency domain is proposed herein to identify the damage of shear connectors in slab-on-girder bridge structures with or without reference data from the undamaged structure. The transmissibility, which is an inherent system characteristic, indicates the relationship between two sets of response vectors in the frequency domain. Measured input force and acceleration responses from hammer tests are analyzed to obtain the frequency response functions at the slab and girder sensor locations by the experimental modal analysis. The transmissibility matrix that relates the slab response to the girder response is then derived. By comparing the transmissibility vectors in the undamaged and damaged states, the damage level of shear connectors can be identified. When the measurement data from the undamaged structure are not available, a study with only the measured response data in the damaged state for the condition assessment of shear connectors is also conducted. Numerical and experimental studies on damage detection of shear connectors linking a concrete slab to two steel girders are conducted to validate the accuracy and efficiency of the proposed approach. The results demonstrate that the proposed method can be used to identify shear connector damages accurately and efficiently. The proposed method is also applied to the condition evaluation of shear connectors in a real composite bridge with in-field testing data.
\end{abstract}

Keywords: Damage Detection; Shear Connectors; Transmissibility; Structural Monitoring; Slab-on-Girder Bridge; Frequency Response Function; 


\section{Introduction}

Structures may deteriorate with time and will continuously accumulate damage during their services due to aging, material deterioration, and natural hazards. The unnoticed and uncorrected anomalies may potentially produce further damage and finally lead to catastrophic structural failures with a huge loss of properties. Therefore the desire to monitor a structure for detecting local damage at an early stage is prevailing throughout the civil engineering community. Dynamic measurements are usually conducted for the condition assessment of civil structures. Generally, vibration-based damage detection methods can be classified into non-model based (direct correlation) and model-based (model updating). With non-model based methods, the measured structural dynamic characteristics are directly compared for the undamaged and damaged states of structures. The measured vibration properties of interest include frequencies [1], mode shapes [2], mode shape curvature [3], flexibility [4], modal strain energy [5], frequency response function [6], etc. On the other hand, the model-based methods require the finite element model of the structure for iterative updating to make the analytical and measured structural vibration properties as closely as possible. One major difficulty of these methods is that an accurate initial finite element model is required for the updating. In fact, many sources of uncertainties that were introduced into the structure during their construction and service stages make it not easy to obtain an accurate finite element model of the intact structure.

Many bridges are built as the slab-on-girder structures. The concrete slab is supported on the concrete or steel girders, and stirrups are embedded in the girders and cast into the slab as shear connectors to link the slab and girders together. The shear connections between the slab and girders are often subjected to serious stressing, overloading, and fatigue. Consequently, damages may involve a deterioration or break of shear connections in some regions of the structure, causing a decrease of the overall rigidity of the composite structure and a reduction of its ultimate resistance [7]. Damage of shear connectors in slab-on-girder bridges will result in shear slippage between the slab and girder, which significantly reduces the load-carrying capacity of bridges. Condition assessment of shear connectors is of great interest and importance to evaluate the structure integrity of this type of bridges.

Xia et al. [8] proposed a local detection method by directly comparing the frequency response functions of simultaneously measured vibrations on the slab and girder. It was found that the local [Type text] 
method gave better identification results than the global methods, since the global modal information is less sensitive to the local damage of shear connectors. The proposed local detection approach was extended to assess the integrity of shear connectors in real slab-on-girder bridges with in-field testing data [9]. Recently, the wavelet based Kullback-Leibler distance [10] and wavelet packet energy [11] have also been proposed for damage identification of shear connectors. Liu and De Roeck [12] proposed a local condition assessment approach to identify the damage location of shear connectors by using the modal curvature and wavelet transform modulus maxima. All of the aforementioned methods are non-model based methods.

The generalized transmissibility matrix for a multi-degrees-of-freedom system in frequency domain was proposed by Ribeiro et al. [13]. The transmissibility matrix indicates the relationship between two sets of response vectors in frequency domain. The generalized transmissibility concept was applied to the structural dynamic response reconstruction in a structure or a substructure in frequency domain [14]. In Refs. [15, 16], a novel structural health monitoring methodology based on the measured transmissibility from the structure was proposed. The transmissibility functions have also been adopted for damage detection [17] using system zeros in the transfer function as the damage indicators. Besides, the transmissibility concept in frequency domain has been used to identify structural modal parameters $[18,19]$, to update finite element models [20], and to identify structural damages [21].

This paper proposes a non-model based damage detection approach with transmissibility in frequency domain to identify the damage of shear connectors in slab-on-girder bridge structures. The transmissibility is an inherent systematic characteristic, which represents the relationship between two sets of response vectors in frequency domain. Measured input force and acceleration responses from hammer tests are analyzed to obtain the frequency response functions at the slab and girder sensor locations by experimental modal analysis, and then to derive the transmissibility that relates the slab response to the girder response. The transmissibility vectors in the undamaged and damaged states of slab-on-girder bridge structures are compared to identify the damage of shear connectors. In case where the measurement data from the undamaged structure are not available, the measured response data in the damaged state only is used for the condition assessment of shear connectors. Numerical and experimental studies of a concrete slab on two steel girders are conducted to validate the correctness and effectiveness of the proposed approach. Finally, the proposed method is applied to [Type text] 
a real composite bridge.

\section{Damage Detection with Existing Methods}

In order to demonstrate the superiority of the proposed method, the commonly used vibration-based methods are also used to identify shear connector conditions. These methods are briefly reviewed here.

\subsection{Coordinate Modal Assurance Criteria}

The Coordinate Modal Assurance Criteria (COMAC) describes the correlation of mode shapes with respect to an individual point over all the modes. For point $q$, the COMAC is defined as [22]

$$
\operatorname{COMAd}\left(\phi^{u}, \phi^{d}, q\right)=\frac{\left(\sum_{i=1}^{n m}\left|\left(\phi_{i}^{u}\right)_{q}\left(\phi_{i}^{d}\right)_{q}\right|\right)^{2}}{\left(\sum_{i=1}^{n m}\left(\phi_{i}^{u}\right)_{q}^{2}\right)\left(\sum_{i=1}^{n m}\left(\phi_{i}^{d}\right)_{q}^{2}\right)}
$$

where $\phi^{u}$ and $\phi^{d}$ are the structural mode shapes in the undamaged and damaged states; $\left(\phi_{i}^{u}\right)_{q}$ and $\left(\phi_{i}^{d}\right)_{q}$ represent the $i$ th mode shape values at point $q$ from the undamaged and damaged structures and $\mathrm{nm}$ is the number of mode shapes involved in the COMAC computation. Normally a low COMAC value indicates a worse correlation between two mode shapes and a possible existence of a damage around the point.

\subsection{Modal Flexibility}

The modal flexibility matrix can be estimated from the measured modal frequencies and mass-normalized mode shapes [23] as

$$
\left.F=\sum_{i}^{n m} \frac{1}{\omega_{i}^{2}}\left\{\phi_{i}\right\} \phi_{i}\right\}^{T}
$$

where $F$ is the flexibility matrix, $\omega_{i}$ the $i$ th modal frequency, $\phi_{i}$ the $i$ th mode shape and $n m$ is the number of mode shapes.

If two sets of measurements, one from the intact structure and another from the damaged structure, are taken and modal parameters have been identified from the measurements, the flexibility [Type text] 
matrix for the two cases can be obtained and its change is calculated as

$$
\Delta=F_{i}-F_{d}
$$

where $F_{i}$ and $F_{d}$ are the flexibility matrices for the intact and damaged cases, respectively. For each measurement location $j$, let $\bar{\delta}_{j}$ be the maximum absolute value of the elements in the corresponding column of $\Delta$, i.e.

$$
\bar{\delta}_{j}=\max _{i}\left|\delta_{i j}\right|
$$

where $\delta_{i j}$ are elements of the $j$ th column of $\Delta$. To detect and locate damages in a structure, the quantity $\bar{\delta}_{j}$ is used as the measure of change in flexibility for each measurement location.

\subsection{Relative Difference of Frequency Response Functions between Slab and Girder}

The abovementioned two methods are global detection methods, which are achieved by comparing the identified modal information from the undamaged and damaged structures. They need measurements from the undamaged structure. A local method has been proposed [9] based on the relative difference of the frequency response function (RDFRF) between the slab and girder from the existing structure only, which is used to evaluate the condition of the shear connectors. The RDFRF is defined as

$$
\operatorname{RDFRF}\left(H_{i}^{G}, H_{i}^{S}\right)=\left\|\left\{H_{\mathrm{i}}^{\mathrm{G}}\right\}|-|\left\{H_{i}^{S}\right\}\right\| / \|\left\{H_{\mathrm{i}}^{\mathrm{G}}\right\}+\left|\left\{H_{i}^{S}\right\}\right| \mid
$$

where, $H_{i}$ is the frequency response function at the $i$ th point; superscript $G$ and $S$ denote that sensor points are on the girder and slab, respectively; $\|\cdot\|$ is the Euclidean norm and $|\cdot|$ is the absolute value (or magnitude of the complex numbers). A high value of RDFRF means a significant difference in the responses at a particular point, which indicates the existence of damage in the vicinity.

\section{Damage Detection of Shear Connectors with Transmissibility}

The damage detection of shear connectors in slab-on-girder bridge structures is conducted with the transmissibility in frequency domain. Two cases are studied in this paper. In the first case, the 
measured responses from the undamaged structure are used as the reference. In the second case, it is assumed that no measurement data from the reference structure are available, which represents a more realistic situation in assessment of existing structures. The generalized transmissibility concept in frequency domain for a multi-degree-of-freedom (DOF) system was proposed by Ribeiro et al. [13]. The transmissibility concept for damage detection of shear connectors for the two cases mentioned above will be briefly reviewed.

\subsection{Frequency Response Function}

The equation of motion of a damped structure with $n$ DOFs can be written as

$$
[M]\{\ddot{x}(t)\}+[C]\{\dot{x}(t)\}+[K]\{x(t)\}=\{F(t)\}
$$

where $M, C$ and $K$ are the $n \times n$ mass, damping and stiffness matrices of the structure, respectively; $\ddot{x}, \dot{x}$ and $x$ are respectively the nodal acceleration, velocity and displacement vectors of the structure; and $\{F(t)\}$ is a vector of applied forces. Rayleigh damping is assumed in the numerical study.

The Fourier transform of Equation (6) gives

$$
\left(-\omega^{2} M+j \omega C+K\right) X(\omega)=F(\omega)
$$

Therefore, the displacement response in frequency domain is given as

$$
X(\omega)=H_{d}(\omega) F(\omega)=\left(-\omega^{2} M+j \omega C+K\right)^{-1} F(\omega)
$$

where $H_{d}(\omega)=\left(-\omega^{2} M+j \omega C+K\right)^{-1}$ is the displacement frequency response function matrix. The frequency response function matrix represents the inherent system frequency response characteristics. It can be reconstructed from the experimental modal analysis, or obtained from the finite element analysis of the structure.

The acceleration response in frequency domain can be obtained from Equation (7) as

$$
\ddot{X}(\omega)=-\omega^{2} X(\omega)=H_{a}(\omega) F(\omega)=-\omega^{2} H_{d}(\omega) F(\omega)
$$

where $H_{a}(\omega)=-\omega^{2} H_{d}(\omega)$ is the acceleration frequency response function matrix. In this study, the acceleration frequency response function is obtained from the experimental modal analysis using the measured input force and output acceleration responses with the DIAMOND toolbox [24] in Matlab 
environment by the rational fraction polynomial method [25].

\subsection{Transmissibility in a Structure in Frequency Domain}

Assuming that $F(\omega)$ is a vector of applied excitation forces on the structure in frequency domain, $\ddot{X}_{1}(\omega)$ is the first set of acceleration response transformed to the frequency domain, and $\ddot{X}_{2}(\omega)$ is the second set of acceleration response in the frequency domain. The following equation can be obtained from Equation (9)

$$
\left\{\begin{array}{l}
\ddot{X}_{1}(\omega)=H_{a}^{1}(\omega) F(\omega) \\
\ddot{X}_{2}(\omega)=H_{a}^{2}(\omega) F(\omega)
\end{array}\right.
$$

where $H_{a}^{1}(\omega)$ and $H_{a}^{2}(\omega)$ denote the sub-matrices of the acceleration frequency response function matrix relating the applied forces to the first and second sets of response vectors, respectively. The following response reconstruction equation can be obtained as

$$
\ddot{X}_{2}(\omega)=T(\omega) \ddot{X}_{1}(\omega)
$$

where $T(\omega)$ is the transmissibility matrix.

$$
T(\omega)=H_{a}^{2}(\omega)\left(H_{a}^{1}(\omega)\right)^{+}
$$

Equation (11) represents the relationship between the first and second sets of response vectors. It should be noted that the number of coordinates in the first set response vector should be at least equal to or greater than the number of applied force coordinates such that a pseudo-inverse $\left(H_{a}^{1}(\omega)\right)^{+}$can be obtained [26]. It is noted that the transmissibility is a vector with scalar numbers at all the frequency lines $\omega$ in the spectrum when only one response in the first set and one response in the second set are available.

\subsection{Damage Detection with Measured Responses of Undamaged Structure}

A local condition assessment approach is proposed based on the fact that when the shear connector is damaged, the behavior of the slab that is not fully connected to the girder may not be exactly the same as that of the girder. The difference in the responses of the slab and girder then can be used to detect the conditions of shear links. When the measured responses from the undamaged structure are 
available, the transmissibility from the slab response to the girder response is used to detect the damage of shear connectors. The reason that the local transmissibility is selected for shear connector condition identification is that it will change significantly as the loading transfer path from the slab to the girder varies due to the damage of shear connectors. Sensors are placed on top of the slab to measure the response of the slab, and underneath the corresponding girder location to record the response of the girder.

The damage index is defined as the relative difference of the magnitude of transmissibility vectors between the undamaged and damaged states

$$
D I\left(T_{u d}, T_{d}\right)=\frac{\left\|\left\{T_{u d}\right\}|-|\left\{T_{d}\right\}\right\| \|}{\left\|\left\{T_{u d}\right\}|+|\left\{T_{d}\right\}\right\|}
$$

where $T_{u d}$ and $T_{d}$ are the transmissibility matrices from the slab response to the girder response in the undamaged and damaged states, respectively. They are represented using Equation (12) as

$$
\left\{\begin{array}{c}
T_{u d}(\omega)=H_{\text {aud }}^{g}(\omega)\left(H_{\text {aud }}^{s}(\omega)\right)^{+} \\
T_{d}(\omega)=H_{a d}^{g}(\omega)\left(H_{a d}^{s}(\omega)\right)^{+}
\end{array}\right.
$$

where $H_{\text {aud }}^{g}(\omega)$ and $H_{\text {aud }}^{s}(\omega)$ are the acceleration frequency response functions corresponding to sensor locations on the girder and slab in the undamaged state, respectively; $H_{a d}^{g}(\omega)$ and $H_{a d}^{s}(\omega)$ are the corresponding frequency response functions in the damaged state. Since there is only one response in each set response vector, e.g. one response in the first set and one response in the second set, respectively, Equation (14) can also be written as

$$
\left\{\begin{array}{c}
T_{u d}(\omega)=H_{a u d}^{g}(\omega) / H_{a u d}^{s}(\omega) \\
T_{d}(\omega)=H_{a d}^{g}(\omega) / H_{a d}^{s}(\omega)
\end{array}\right.
$$

It can be seen that the transmissibility vectors $T_{u d}(\omega)$ and $T_{u d}(\omega)$ are two complex vectors at all frequency lines. A high damage index value in Equation (13) denotes a significant difference in the transmissibility at a specific location and indicates the existence of damage of shear connector in the nearby area. The proposed damage detection method is cataloged as the non-model based method as the finite element model of the structure is not required in the identification. 


\subsection{Damage Detection without Measured Responses of the Undamaged Structure}

In practice usually no measurement data from the reference model is available. In this case, only the responses from the structure in its current state can be measured and used for damage identification. In our previous study [9], a damage index based on the RDFRF between the vibration signals measured simultaneously on bridge deck and girder was proposed. The advantage of the method is that it does not need any data on a reference model. The drawback of the method is that although the proposed damage index is based on the vibration measurement and sensitive to shear link damage, its sensitivity range is limited to a radius of about $1 \mathrm{~m} \mathrm{[9].} \mathrm{In} \mathrm{other} \mathrm{words,} \mathrm{shear} \mathrm{link} \mathrm{damage} \mathrm{can} \mathrm{be}$ reliably identified only when the measurement location is within a $1 \mathrm{~m}$ distance from the damaged location. In the present paper, a new technique and damage index based on vibration transmissibility are proposed. The transmissibility reflects the local change and increases the sensitivity in identifying the damage of shear connectors.

To perform the damage detection with only the measured responses in the current state, a reference sensor is defined here to compute the transmissibility vectors from this reference sensor location to a slab sensor location and the corresponding girder sensor location, respectively. These transmissibility vectors can be expressed as

$$
\left\{\begin{array}{l}
T_{s r}(\omega)=H_{a d}^{s}(\omega) / H_{a d}^{r}(\omega) \\
T_{g r}(\omega)=H_{a d}^{g}(\omega) / H_{a d}^{r}(\omega)
\end{array}\right.
$$

where $H_{a d}^{r}(\omega)$ is the acceleration frequency response function at the reference sensor location in the damaged state.

The frequency response functions $H_{a d}^{r}(\omega)$ at the reference sensor location in the first and second rows of Equation (16) are the same. If the damage of shear connectors is present, the frequency response function at the slab sensor location $H_{a d}^{s}(\omega)$ will be different from that at the girder $H_{a d}^{g}(\omega)$. Therefore, the transmissibility from the reference sensor to the slab sensor $T_{s r}(\omega)$ should not be exactly the same as the transmissibility from the reference sensor to the girder sensor $T_{g r}(\omega)$. A damage index is then defined based on this fact for identifying the damage of shear connectors as 


$$
D I\left(T_{s r}, T_{g r}\right)=\frac{\|\left\{T_{s r}\right\} \mid-\left\{\left\{T_{g r}\right\}\|\|\right.}{\left\|\left\{T_{s r}\right\} \mid+\left\{T_{g r}\right\}\right\|}
$$

A high damage index value from Equation (17) indicates the significant difference between the two transmissibility vectors and thus the existence of damage of shear connectors nearby.

\subsection{Establishment of a Threshold Value for Damage Detection}

When $n$ damage indices are obtained at different sensor locations, the mean value and standard deviation of these damage indices can be computed and expressed as $\mu$ and $\sigma$. The one-side upper confidence limit for the damage index can be obtained as [27]

$$
U L=\mu+Z_{\alpha} \times \frac{\sigma}{\sqrt{n}}
$$

where $Z_{\alpha}$ is the value of a standard norm distribution with zero mean and unit standard deviation such that the cumulative probability is $100 \times(1-\alpha) \%$. The upper confidence limit $U L$ is considered as a threshold value to indicate possible abnormalities in the damage index. The definition of this threshold value is based on the statistical properties of the calculated damage indices from measured responses. Damage index values larger than the threshold value indicate the existence of damage, and those smaller than the threshold value are identified as undamaged. In this regard, a new damage indicator $(D I-U L)$ is used in this study to identify the occurrence of damage, in which $D I$ is the damage index obtained from Equations (13) or (17). It should be noted that the measured locations are identified to be undamaged when the damage index $D I$ is less than the threshold value. To highlight the damage locations detected, the values of $(D I-U L)$ on undamaged locations are taken as zeros and positive values of $(D I-U L)$ on possibly damaged locations will be shown.

\section{Numerical studies}

Numerical studies on a simply-supported slab-on-girder structure are conducted to demonstrate the accuracy and efficiency of the proposed approaches for damage detection of shear connectors in composite bridges. Figure 1 shows the plan view and cross section of the structure and details of a 
shear connector. The concrete slab is placed on two steel I-type girders, and shear connectors are used to link the slab and girders together. Each girder has 16 shear connectors with equal space and there are 32 shear connectors in total in the structure. They are denoted as SC1 SC32 in Figure 1(a). The cross-section of the structure is shown in Figure 1(b). To be consistent with the experimental study that will be described later, the shear connector is simulated as a metric bolt screwing into a metal nut, as shown in Figure 1(c). The shear link fixity is provided by securing the metric bolt. When the bolt is unscrewed from the metal nut, it represents the damage of the corresponding shear connector.

\subsection{Finite Element Model and Sensor Placement Configuration}

Slab and steel girders are modeled with shell elements, and shear connectors are modeled with beam elements [9] that link the slab and girders. The axial stiffness and shear stiffness of a shear connector are obtained by the formulas in an existing study [28]. The finite element model of the slab-on-girder structure consists of 695 nodes, 600 shell elements and 32 beam elements. Each node has six DOFs and the system has 4170 DOFs in total. The Young's modulus and mass density of the slab concrete are $3.18 \times 10^{4} \mathrm{MPa}$ and $2500 \mathrm{~kg} / \mathrm{m}^{3}$, respectively, and those of the steel girder are $2 \times 10^{5} \mathrm{MPa}$ and $8092 \mathrm{~kg} / \mathrm{m}^{3}$, respectively. The first three natural frequencies of the undamaged structure in the vertical direction are $35.74 \mathrm{~Hz}, 109.94 \mathrm{~Hz}$ and $144.76 \mathrm{~Hz}$, respectively. Rayleigh damping is assumed and the damping ratios for the first two modes are taken as $\xi=0.012$.

Figure 2 shows that accelerometers are placed on the slab, denoted as "SA1-SA8" and "SB1-SB8", and underneath the girders, denoted as "GA1-GA8" and "GB1-GB8", to measure the acceleration responses from impact tests. Two damage scenarios are assumed, as shown in Table 1. Damage of shear connectors are simulated by fully removing the specific shear links (represented by beam elements in the finite element model) to simulate the failure of blots. Measured responses from the structure in both the undamaged and damaged states are obtained. 8192 points of measured input and response data with a sampling rate of $1000 \mathrm{~Hz}$ are used to analyze the frequency response functions with DIAMOND toolbox. The obtained frequency response functions at slab and girder sensor locations are used to calculate the transmissibility.

To simulate the effect of measurement noise, a normally distributed random noise with zero mean and specific standard deviation is added to the calculated dynamic response as [Type text] 


$$
\ddot{x}_{n}=\ddot{x}_{c a l}+E_{p} N_{\text {oise }} \operatorname{std}\left(\ddot{x}_{c a l}\right)
$$

where $\ddot{x}_{n}$ and $\ddot{x}_{c a l}$ are the simulated response with noise effect and the original calculated response, respectively; $E_{p}$ is the noise level and equals to 0.03 if $3 \%$ noise is included in the response; $N_{\text {oise }}$ is a standard normal distribution vector; and $\operatorname{std}\left(\ddot{x}_{c a l}\right)$ denotes the standard deviation of the original calculated response. Two noise levels (3\% and 5\%) are considered in the measured acceleration responses and $1 \%$ noise in the hammer excitation force in this study.

\subsection{Modal Analysis Results}

Natural frequencies and the identified four vertical mode shapes of the structure in the undamaged and damaged states from responses without noise are identified and the modal frequencies are shown in Table 2. Modal Assurance Criterion (MAC) values of these mode shapes at the slab sensor locations of two damage scenarios are shown in Table 3. It can be seen that the changes of the identified frequencies are not prominent in damage Scenario 1. The largest one is $3.2 \%$ in the second frequency, indicating it could be difficult to confidently identify the damage with the changes of frequencies. For damage Scenario 2, the change of the first frequency is $5.48 \%$ and it is up to $13.1 \%$ in the fourth frequency. MAC value of the fourth mode shape is 0.36 . These significant changes are observed because the shear connectors at the two support locations are removed and this structure has no diaphragms at the two ends. Therefore the damage of shear connectors at the two support locations induces large changes of frequencies and MAC values. Shear forces of a simply-supported structure at the two support locations are generally larger than those at other places, therefore it is possible and practical that the damages of shear links are presented near the supports. These significant changes indicate the damage existence could be confidently identified with global modal information. However, the damage location cannot be identified without performing further analysis such as model correlation and updating.

\subsection{Case A: Damage Detection with Measured Responses of Undamaged Structure}

In this case, acceleration response data and hammer impact force from the structure in both the undamaged and damaged states are measured to identify the modal information, including 
frequencies, mode shapes and the frequency response functions for damage detection.

\subsubsection{Damage Detection with Global Modal Information}

Damage detection results with COMAC based on the identified four mode shapes are shown in Figure 3. It can be seen that COMAC fails to identify the damages in Scenario 1 as no obvious reductions in COMAC values are observed at the sensor location No. 5 in both girders. For Scenario 2, COMAC can identify the damages in sensor locations No. 1 and No. 8 of Girder A. However, two false identifications are detected at sensor locations No. 1 and No. 8 of Girder B as they are located in the opposite girder of the true damaged locations, and two more false identifications are observed at sensor location No. 4 in the middle of both girders. Figure 4 shows the damage detection results with changes in flexibility. It can be found that the damages in sensor location No. 5 of Scenario 1 can be detected, while two false identifications are observed at sensor location No. 6 in both girders as they are adjacent to the true damage locations. The damages simulated in Scenario 2 are identified accurately indicating that the detection using changes in flexibility is more sensitive than COMAC by comparing Figures 3 and 4. However, the detection results with global modal information from noisy responses can be much worse with more possible false identifications.

\subsubsection{Damage Detection with Transmissibility}

The transmissibility vectors in the frequency range of $25 \sim 125 \mathrm{~Hz}$ in the undamaged and damaged states are used to calculate the damage index. This frequency range covering only the first two modes is defined to investigate the reliability and robust of the proposed damage detection approach. $\alpha$ is set to be 0.02 in Equation (18) to obtain the upper confidence limit and then compute the damage index $(D I-U L)$ with consideration of this threshold value. Figure 5 shows the damage detection results from measurements without noise effect. The identified results of Scenario 1 for girders A and B are shown in Figures 5(a). It can be clearly seen that higher damage indices at the sensor location No. 5 close to the damaged shear connectors in both girders are observed. Figure 5(b) shows the detection results of Scenario 2 in girders A and B. It can be seen that damage index values at sensor locations No. 1 and No. 8 in girder A are around 0.2, which indicates that the damage of shear connectors are presented in these sensor locations. These identified locations matches well with the 
introduced damage locations. However, it should be noted that the damage index at sensor location No.4 in girder $\mathrm{A}$ is also relatively high and around 0.05. This may lead to a false identification because the response at the mid span of the simply-supported model is the largest. The steel girder and concrete slab in the current structure model are only connected by 16 shear connectors between each girder and slab. Some difference in responses on slab and girder is expected, and is most significant when the response is relatively large at the mid span. This response difference is also picked up by the proposed damage index. Nonetheless the proposed method successfully identified all the simulated shear connector damages, which are not likely to be detected with visual inspections because shear connectors are buried inside the structures, and might not be able be identified accurately with other vibration-based parameters such as frequencies, mode shapes and flexibility as demonstrated above.

Figure 6 shows the detection results from the simulated data with smeared noise. The damage index values in Scenario 1 at sensor location No.5 in both girders A and B are around 0.15 in two noise cases, indicating the existence of damage of shear connectors in the nearby area. Damage index values in Scenario 2 at sensor locations No.1 and No.8 in girder A are around 0.2 in two noise cases. The introduced damages of shear connectors in both Scenarios 1 and 2 are identified correctly even the vibration signals are smeared with noise. The robustness of the proposed approach for damage detection of shear connectors from noisy measurements is demonstrated. However, similar to the results shown in Figure 5(b), a false identification at sensor location No.4 in girder A occurs in Scenario 2. The damage index value at this location increases with the noise level and is up to 0.1 when the noise level is $5 \%$.

Detection results with COMAC and changes in flexibility shown in Figures 3 and 4 demonstrate that using global modal information may not correctly identify the damages of shear connectors in minor damage case with very small frequency and MAC changes. The proposed local dynamic condition assessment approach can successfully identify the shear connector damage locations in two damage scenarios and shows the advantages over the traditional detection method based on global modal information with more accurate results and less false identifications. A few false identifications occur at the mid span owing to relatively large displacement response at this location since the steel girders and concrete slab are not continuously connected together in the model considered in this study.

[Type text] 


\subsection{Case B: Damage Detection without Measured Responses of the Undamaged Structure}

Damage detection of shear connectors with only the measured data from the damaged structure is conducted. The detection is conducted with both the method in a previous study [9] and the proposed approach, and a comparison is made to demonstrate the performance and advantages of the proposed approach.

\subsubsection{Damage Detection with Relative Difference of Frequency Response Functions}

The relative difference between frequency response functions on the slab and corresponding girder locations is used to detect the damage of shear connectors. Figures 7 and 8 show the damage detection results based on the relative difference of frequency response functions without and with noise effect, respectively. The introduced damages are identified correctly with several false identifications on the close sensor locations, such as sensor location No.4 in Scenario 1 and sensor locations No.1 and No.8 of Girder B in Scenario 2.

\subsubsection{Damage Detection with Transmissibility}

One more sensor location is added in the sensor placement configuration described in Section 4.3.2. It is placed at the central slab location on the top of girder A and defined as the reference sensor, as shown in Figure 2. The same damage scenarios are defined. The transmissibility vectors from the reference sensor response to the slab and girder responses in the damaged state respectively are obtained from Equation (15), and the transmissibility vectors in the frequency range of $25 \sim 125 \mathrm{~Hz}$ are used to compute the damage index in Equation (17). $\alpha$ is set to be 0.02 to obtain the upper confidence limit. Figures 9(a) and 9(b) show the identified results of damage Scenarios 1 and 2 from the measured responses without noise, respectively. It can be clearly seen that the introduced damages of shear connectors are identified accurately in both damage scenarios. Several very small false identifications are observed and their damage index values are less than 0.05 , such as sensor location No.4 in girder A in Scenario 1 and sensor location No.8 in girder B in Scenario 2. They are close to the true damage locations and wrongly identified due to the smearing effect in the damage detection. Figure 10 shows the damage detection results from simulated measured responses with noise. The identified results demonstrated that the locations of damages in both scenarios are detected accurately. [Type text] 
The damage index values in Scenario 1 at sensor locations No.5 in both girders are around 0.15 in two noise cases. The identified damage indices in Scenario 2 at sensor locations No.1 and No.8 in girder A are around 0.3. Several false identifications at sensor location No.4 in girder A in Scenario 1 and No.8 in girder B in Scenario 2 are observed and their damage index values increase up to 0.05 with 5\% noise included in the responses.

\section{Experimental Validation}

\subsection{Experimental Setup}

Experimental studies are conducted to validate the proposed damage detection approach. A slab-on-girder structure fabricated in the laboratory provided the measured acceleration responses with measurement and environmental noises for the damage detection. The performance of the proposed approach for damage identification of shear connectors is investigated.

A structural model with a concrete slab supported on two steel girders was designed and constructed. Sixteen shear connectors were equally mounted in each girder linking the slab and steel girder together. The bridge was located on two steel frames which were fixed on the strong ground as shown in Figure 11. The design of shear connectors considers the ability not only to simulate failure of specific shear links, but also to reset-up them to the undamaged state. Therefore, a metric bolt screwing into a metric nut connecting the slab and girder was simulated as the shear connectors. The metric nuts were welded onto the reinforcement bar in the slab before pouring. Design and setup of shear connectors can be seen in Figure 12. If all the bolts are screwed into the nuts, the structural condition corresponds to the undamaged state. The damage of shear connectors is introduced into the structure by fully removing some of the bolts to simulate the failure of blots. The dimensions of the laboratory model are the same as those of the model in the numerical study. It should be noted that since the proposed damage detection approach for shear connectors is non-model based, the finite element model of the structure is not required. Therefore the dimensions and material properties of the slab, girder and shear connectors are not required for identification.

Nine Kistler 8330A3 accelerometers were used in the laboratory dynamic tests to collect the acceleration responses of the structure. A sixteen-channel conditioner and data acquisition system was employed to record the signals. An instrumented 5820A sledge hammer with a rubber tip was 
used to generate the impact excitation. Figure 13 shows the numbering of shear connectors and sensor locations defined in the testing model. One sensor is placed on the reference location and other eight sensors were repeatedly placed on the slab or underneath the girders to measure the responses at sensor locations SA1 SA8, SB1 SB8, GA1 GA8 and GB1 GB8. Two damage scenarios are listed in Table 4. Excitation with hammer impact was applied at the left quarter span of the slab as shown in Figure 13. The measuring sampling frequency was set as $2000 \mathrm{~Hz}$. DIAMOND data analyzing software was employed to conduct the experimental modal analysis and obtain the frequency response function at each sensor location.

\subsection{Identified Frequencies and Mode Shapes}

The first three identified natural frequencies of the structure in the undamaged and damaged states are listed in Table 5. Figure 14 shows the corresponding identified mode shapes of the undamaged structure. The identified mode shapes of the damaged structure are similar and not shown in this paper due to the page limit. MAC values of three mode shapes at the slab sensor locations of two damage states are shown in Table 6. It can be seen from Table 5 that the minor changes of the first three frequencies are observed in damage Scenario 1. The largest one is $0.82 \%$ at the third frequency, and its corresponding MAC value is 0.923 . Therefore it could be difficult to identify the damage with the changes of frequencies and mode shapes. For the damage Scenario 2, the largest change of natural frequency is $17.08 \%$ at the third mode, and its MAC value is 0.449 . This observation is consistent with those in the modal analysis of numerical studies with same explanations.

\subsection{Case A: Damage Detection with Measured Responses of Undamaged Structure}

\subsubsection{Damage Detection with Global Modal Information}

Figure 15 shows that COMAC fails to identify the damages in Scenarios 1 and 2 indicating that COMAC is not a good indicator to detect the local damage of shear connectors because the identified frequency and mode shapes would be polluted by the noise effect in lab testing and not easy to locate the local change of shear link conditions. Figure 16 shows the damage detection results with changes in flexibility. It can be found that the damages in Scenario 1 are not detected correctly, while damages in Scenario 2 are identified accurately indicating that the detection using change in flexibility is better than COMAC but not applicable for the minor damage case with smaller modal information changes. [Type text] 


\subsubsection{Damage Detection with Transmissibility}

The same 16384 recorded data points were used to perform the signal analysis to obtain the frequency response function. Hanning window was used to reduce the leakage in the fast Fourier transform. Transmissibility vectors in the undamaged and damaged states were calculated from Equation (15). Figure 17 shows the Fourier spectrum of the measured response at sensor location SB5 from damage Scenario 1. It is shown that the magnitude of Fourier spectrum after $400 \mathrm{~Hz}$ is very small. Therefore the transmissibility matrices in the frequency range $15 \mathrm{~Hz} \sim 400 \mathrm{~Hz}$ in the undamaged and damaged states are used to compute the damage index and identify the damage location of shear connectors. Since more uncertainties may exist in the experimental testing, $\alpha$ is set as 0.05 such that the cumulative probability of the upper confidence limit is $95 \%$.

Figures 18(a) and 18(b) show the identified results of Scenarios 1 and 2, respectively. It can be found from Figure 18(a) that two damage index values at the sensor location No.4 in two girders are observed indicating that the damage of shear connectors presented in this area in Scenario 1. This observation illustrates that the introduced damage of SC7, SC8, SC23 and SC24 can be identified correctly. It should be noticed that the damage index at the sensor location No.4 in girder B is around 0.15 , while it is very small at sensor location No.4 in girder A although the damage severity at the two locations is the same. This may be because the residual friction forces between the concrete and the steel girder in these two locations are not exactly the same. A larger residual friction force may exist in sensor location No.4 of girder A, making the difference between the responses on the slab and underneath the girder not significant. This observation indicates that it is difficult to use damage index value to quantify the damage. This limitation is the same as other non-model based methods, i.e., they can locate the damage but very difficult to quantify the damage. Damage detection results for Scenario 2 in girders A and B are shown in Figure 18(b). It can be found that these two damage locations are identified accurately. The damage index at sensor location SA1 is around 0.6, while its value at SA8 is around 0.05 . This is similar to the observations of Scenario 1 with the same possible explanation. 


\subsection{Case B: Damage Detection without Measured Responses of the Undamaged Structure}

\subsubsection{Damage Detection with Relative Difference of Frequency Response Functions}

Figure 19 shows the damage detection results with the relative difference of frequency response functions between slab and girder. Measured acceleration and hammer impact force data are used to extract the frequency response functions in the damaged state for identification. The damages in both scenarios are identified, while with several false identifications, for example, sensor locations No.3 and No.8 of Girder B in Scenario 1, sensor location No.5 of Girder B in Scenario 2.

\subsubsection{Damage Detection with Transmissibility}

When only the measured responses from the damaged structures are available, the transmissibility vectors from a reference sensor response to a slab response and a girder response respectively can be computed and used to derive the damage index with Equation (16). A reference sensor location is defined as shown in Figure 13 in this study. Figure 20 shows the magnitude of the transmissibility from reference sensor response to slab response at sensor location SA1 in damage Scenario 1. It can be seen that this transmissibility covers all the frequency range with same order of magnitudes. Therefore, transmissibility matrices in the frequency range of $15 \mathrm{~Hz} \sim 1000 \mathrm{~Hz}$ are used to compute the damage index. Figures 21(a) and 21(b) show the damage detection results from the measured responses in the damaged states for Scenarios 1 and 2, respectively. It can be seen that the introduced damages of shear connectors in both damage scenarios are identified accurately. A small false identification is observed at sensor location No.7 in girder B in Scenario 1. The identified results in experimental studies demonstrate that the locations of damages in both scenarios are detected accurately with only the measured responses from the damaged structure.

Experimental studies demonstrate that the proposed damage detection approach with transmissibility in frequency domain can identify the damage locations of shear connectors accurately and efficiently with and without reference data from the undamaged structure. Comparing the results shown in Figures 19 and 21 from the method in Ref. [9] and the proposed approach, better detection results are given with less false identifications. 


\section{In-field Testing}

The proposed damage detection method is further applied to evaluate the shear link conditions of a real bridge. Bridge No.852 is located on North West Coastal Highway over the Balla Balla River in the Shire of Roebourne, Western Australia. It is a pre-streesed concrete bridge constructed in 1975, which consists of three spans with an overall length of $53.95 \mathrm{~m}$ and width of $9.398 \mathrm{~m}$. The central span of the bridge is $18.288 \mathrm{~m}$ and the external spans are $17.831 \mathrm{~m}$. The deck of the bridge is the cast-in-situ reinforced concrete slabs supported by seven precast pre-stressed I-type girders, as shown in Figure 22. The shear connectors are used to link the slab and girders together. They are $12 \mathrm{~mm}$ in diameter and enter the RC slab for a 100mm length before being bent for anchorage. Spacing of the connectors varies from $76 \mathrm{~mm}$ in the ends to $381 \mathrm{~mm}$ in the center of the girders. More details about the design of the bridge are referred to a technical report [29].

Field dynamic testing on this bridge was carried out in 2005 [29]. These data are analyzed in this study to identify shear link conditions with only the measurements from the existing structure. Figure 23 shows the sensor placement on the slab and girders. The bridge has three spans each with seven girders, and so accelerometers were placed on the slab locations corresponding to the seven girders, allowing comparison with the underneath girder measurements. There are nineteen sensor locations on the slab along the longitudinal direction in each row. Site condition and in-field testing safety concerns make the measurement points underneath the girders limited to the sensor locations shown in Figure 23(b). The girders are numbered as A, B, C, D, E, F and G, respectively. An instrumented DYTRAN 5803A sledge hammer was used in the impact tests, and eight Kistler accelerometers were repeatedly employed to measure the acceleration responses on slab and girders. The sampling rate was set to $100 \mathrm{~Hz}$, and 1024 data points were recorded in each impact.

Frequency response functions were extracted from the measured acceleration response data and hammer impact force with DIAMOND toolbox. Frequency range from $1 \sim 21 \mathrm{~Hz}$ is used to include the first ten modes [29]. Five reference sensors, e.g. A10, B10, C10, D10, E10 and F10 are defined to average the damage index. Figure 24 shows the identification results based on the differences of frequency response functions and transmissibility vectors. Damages of shear connectors from both methods are mostly identified at the support locations, especially near the abutments 1 and 2 as the shear forces at the supports are generally larger than those at other locations. The condition 
assessment results are reasonable since the shear connections at the support locations are more easily and likely to be damaged.

\section{Conclusions}

This paper presents a dynamic condition assessment approach with transmissibility in frequency domain to identify the damage of shear connectors in slab-on-girder bridge structures. The method can be used to detect shear connector damages with or without reference measurement data of the undamaged structure. Numerical and experimental studies are conducted to demonstrate the accuracy and efficiency of the proposed approaches. Both numerical and experimental results demonstrated that the proposed approaches can reliably identify shear connector damages and outperforms the traditional global modal-based methods to detect shear link damages. The proposed approach gives detection results with less false identifications. The proposed method is also used to detect shear link conditions in a real bridge. Both the proposed method and local method based on the difference in frequency response functions predict the similar shear connector conditions of the bridge, i.e., possible shear connector damages exist near the supports of the three-span continuous bridge.

\section{ACKNOWLEDGEMENT}

The work described in this paper was supported by "Supporting Infrastructure Management by Combining Sensors and Asset Information Models - Project No. 3104", Australia Cooperative Research Centre for Infrastructure and Engineering Asset Management (CIEAM-II). Thanks go to final-year students in the School of Civil and Resource Engineering at The University of Western Australia, Zac Lampitoc and Adina Lieblich for their help to construct the laboratory model. 


\section{References:}

[1] Z. Wang, F. T. K. Au and Y. S. Cheng, Statistical damage detection based on frequencies of sensitivity-enhanced structures, Int. J. Struct. Stab. \& Dyn, 8(2) (2008) 231 - 255.

[2] R. Rodriguez-Rocha, F. J. Rivero-Angeles and E. Gomez-Ramirez, Application of the baseline stiffness method for damage detection of an RC building without baseline modal parameters utilizing the independent component analysis for modal extraction, Int. J. Struct. Stab. \& Dyn, 13(3) (2013) 1250074.

[3] A. K. Pandey, M. Biswas, and M. M. Samman, Damage detection from changes in curvature mode shapes, J. Sound. Vib, 145(2) (1991) 321 - 332.

[4] V. A. Tran, S. T. Quek and W. H. Duan, Sensor validation in damage locating vector method for structural health monitoring, Int. J. Struct. Stab. \& Dyn, 11(1) (2011) 149 - 180.

[5] V. Srinivas, C. A. Jeyasehar and K. Ramanjaneyulu, Computational methodologies for vibration-based damage assessment of structures, Int. J. Struct. Stab. \& Dyn. (Online ready).

[6] T. K. Lin, S. L. Hung and C. S. Huang, Detection of damage location using a novel substructure-based frequency response function approach with a wireless sensing system, Int. J. Struct. Stab. \& Dyn, 12(4) (2012) 1250029.

[7] M. Dilena, and A. Morassi, Experimental modal analysis of steel concrete composite beams with partially damaged connection, J. Vib. Control, 10(6) (2004) 897 - 913.

[8] Y. Xia, H. Hao and A. J. Deeks, Dynamic assessment of shear connectors in slab-girder bridges, Eng. Struct, 29(7) (2007) 1457 - 1486.

[9] Y. Xia, H. Hao, A. J. Deeks, and X. Q. Zhu, Condition assessment of shear connectors in slab-girder bridges via vibration measurements, J. Bridge. Eng. ASCE, 13(1) (2008) 43 - 54.

[10] X. Q. Zhu, H. Hao, B. Uy, Y. Xia and O. Mirza, Dynamic assessment of shear connection conditions in slab-girder bridges by Kullback-Leibler distance, Adv. Struct. Eng, 15(5) (2012) $771-780$.

[11] W. X. Ren, Z. S. Sun, Y. Xia, H. Hao and A. J. Deeks, Damage identification of shear connectors with wavelet packet energy: laboratory test study, J. Struct. Eng. ASCE, 134(5) (2008) 832 - 841.

[12] K. Liu and G. De Roeck, Damage detection of shear connectors in composite bridges, Struct. Health. Monit, 8(5) (2009) 345 - 356. 
[13] A. M. R. Ribeiro, J. M. M. Silva and N. M. M. Maia, On the generalisation of the transmissibility concept, Mech. Syst. Signal Process, 14(1) (2000) 29 - 35.

[14] S. S. Law, J. Li and Y. Ding, Structural response reconstruction with transmissibility concept in frequency domain, Mech. Syst. Signal Process, 25(3) (2011) 952 - 968.

[15] K. Worden and G. Manson, Experimental validation of a structural health monitoring methodology: part I. novelty detection on a laboratory structure, J. Sound. Vib, 259(2) (2003) $323-343$.

[16] G. Manson and K. Worden, Experimental validation of a structural health monitoring methodology. part II. novelty detection on a gnat aircraft, J. Sound. Vib, 259(2) (2003) 345 - 363.

[17] T. J. Johnson and D. E. Adams, Transmissibility as a differential indicator of structural damage, J. Vib. Acoust. ASME, 124(4) (2002) 634 - 641.

[18] C. Devriendt and P. Guillaume, The use of transmissibility measurements in output-only modal analysis, Mech Syst Signal Process, 21(7) (2007) 2689 - 2696.

[19] C. Devriendt and P. Guillaume, Identification of modal parameters from transmissibility measurements, J. Sound. Vib, 314(1-2) (2008) 343 - 356.

[20] G. Steenackers, C. Devriendt and P. Guillaume, On the use of transmissibility measurements for finite element model updating, J. Sound. Vib, 303(3) (2007) 707 - 722.

[21] J. Li, S. S. Law and Y. Ding, Substructure damage identification based on response reconstruction in frequency domain and model updating, Engineering Structures, 41 (2012) 270 $-284$.

[22] N. A. J. Lieven and D. J. Ewins, Spatial correlation of mode shapes, the co-ordinate Modal Assurance Criterion (COMAC), Proceedings of the 6th International Modal Analysis Conference, 1 (1998) 690-695.

[23] A. K. Pandey and M. Biswas, Damage detection in structures using changes in flexibility, $J$. Sound. Vib, 169(1) (1994) 3-17.

[24] S.W. Doebling, C.R. Farrar, P.J. Cornwell, DIAMOND: a graphical interface toolbox for comparative modal analysis and damage identification, Proceedings of the Sixth International Conference on Recent Advances in Structural Dynamics, Southampton, 1997.

[25] D. J. Ewins, Modal testing: theory, practice and application (Research Studies Press Ltd,. Hertfordshire, U.K., 2000).

[Type text] 
[26] R. Penrose, A Generalized Inverse for Matrices, Proc Cambridge Philos Soc, 51 (1955) 406 413.

[27] S. M. Ross, Introduction to probability and statistics for engineers and scientists, Third edition, (Elsevier Academic Press, USA, 2004).

[28] M. Chiewanichakorn, A. J. Aref, S. S. Chen and S. Ahn, Effective flange width definition for steel-concrete composite bridge girder, J. Struct. Eng. ASCE, 130(12) (2004) 2016-2031.

[29] Y. Xia, H. Hao, G. Zanardo and M. Nguyen, Vibration-based damage detection of shear connectors in Nickol River bridge and Balla Balla River bridge, Part 1: preliminary study, Technical Report, ST-05-01, School of Civil and Resource Engineering, University of Western Australia (2005). 
Table 1 - Damage scenarios in numerical study

Damage scenario Shear connectors removed

\begin{tabular}{lc}
\hline Scenario 1 & SC9, SC10, SC25 and SC26 \\
\hline Scenario 2 & SC1, SC2, SC15 and SC16 \\
\hline
\end{tabular}

Table 2 - Frequencies of the undamaged and damaged structures in numerical study

\begin{tabular}{cccccc}
\hline Mode & Undamaged (Hz) & $\begin{array}{c}\text { Damage } \\
\text { Scenario 1 }(\mathrm{Hz})\end{array}$ & Change (\%) & $\begin{array}{c}\text { Damage } \\
\text { Scenario 2 }(\mathrm{Hz})\end{array}$ & Change (\%) \\
\hline 1 & 35.74 & 35.55 & 0.53 & 33.78 & 5.48 \\
\hline 2 & 109.94 & 106.42 & 3.2 & 101.27 & 7.89 \\
\hline 3 & 144.76 & 143.13 & 1.13 & 142.77 & 1.37 \\
\hline 4 & 233.5 & 228.9 & 1.97 & 202.91 & 13.1 \\
\hline
\end{tabular}

Table 3 - MAC values of the damaged structure in numerical study

\begin{tabular}{ccc} 
Mode & Damage Scenario 1 & Damage Scenario 2 \\
\hline 1 & 1.000 & 0.96 \\
\hline 2 & 0.999 & 0.63 \\
\hline 3 & 0.992 & 0.59 \\
\hline 4 & 0.940 & 0.36 \\
\hline
\end{tabular}


Table 4 - Damage scenarios in experimental study

\begin{tabular}{cc}
\hline Damage scenario & Shear connectors removed \\
\hline Scenario 1 & SC7, SC8, SC23 and SC24 \\
\hline Scenario 2 & SC1, SC2, SC15 and SC16 \\
\hline
\end{tabular}

Table 5 - Identified frequencies of the undamaged and damaged structures in experimental study

\begin{tabular}{cccccc}
\hline Mode & Undamaged (Hz) & $\begin{array}{c}\text { Damage } \\
\text { Scenario 1 }(\mathrm{Hz})\end{array}$ & Error (\%) & $\begin{array}{c}\text { Damage } \\
\text { Scenario 2 (Hz) }\end{array}$ & Error (\%) \\
\hline 1 & 41.27 & 41.12 & 0.36 & 38.05 & 7.80 \\
\hline 2 & 116.61 & 116.52 & 0.08 & 100.35 & 13.94 \\
\hline 3 & 265.54 & 263.37 & 0.82 & 220.19 & 17.08 \\
\hline
\end{tabular}

Table 6 - MAC values of the damaged structure in experimental study

\begin{tabular}{ccc} 
Mode & Damage Scenario 1 & Damage Scenario 2 \\
\hline 1 & 1.000 & 0.984 \\
\hline 2 & 0.999 & 0.898 \\
\hline 3 & 0.923 & 0.449
\end{tabular}




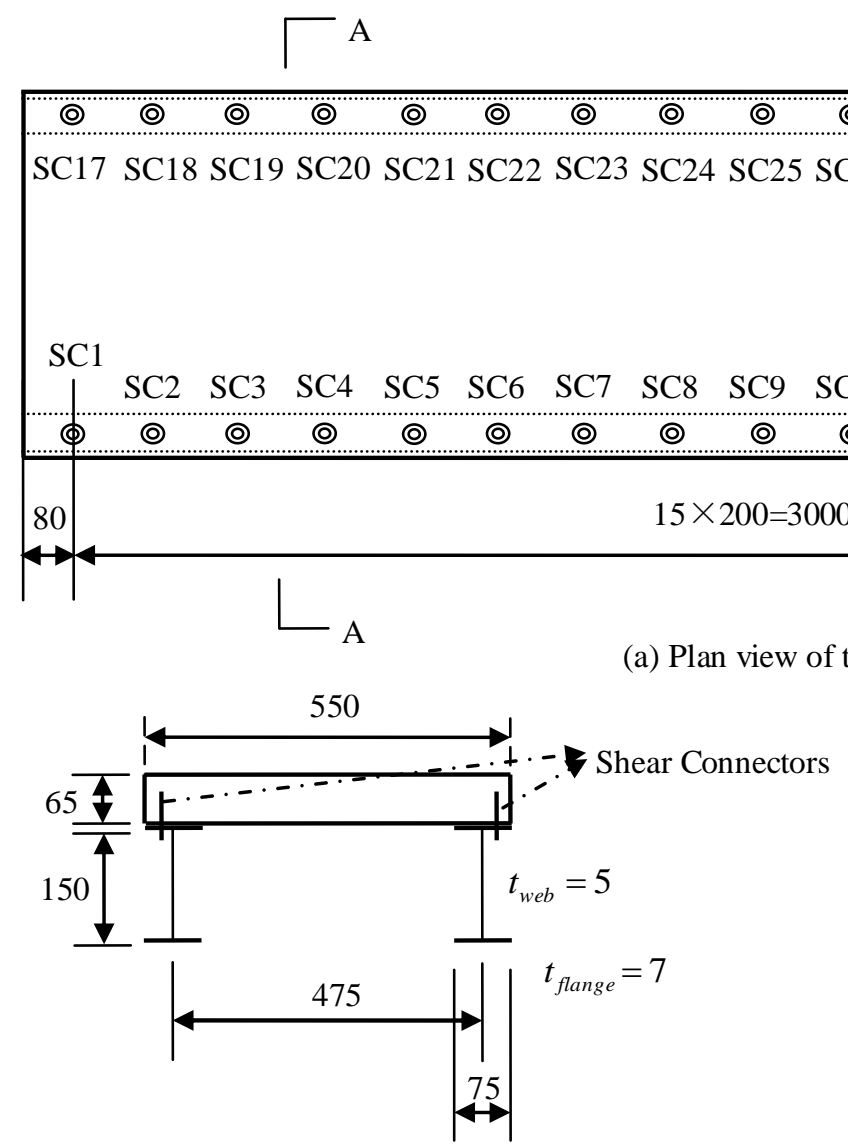

(b) Cross section of the model (A-A)

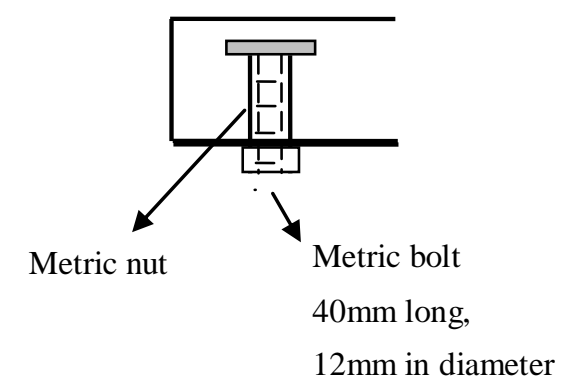

(c) Shear connector

Figure 1 - Dimensions and shear connector details of the slab-on-girder structure (unit: $\mathrm{mm}$ ) 


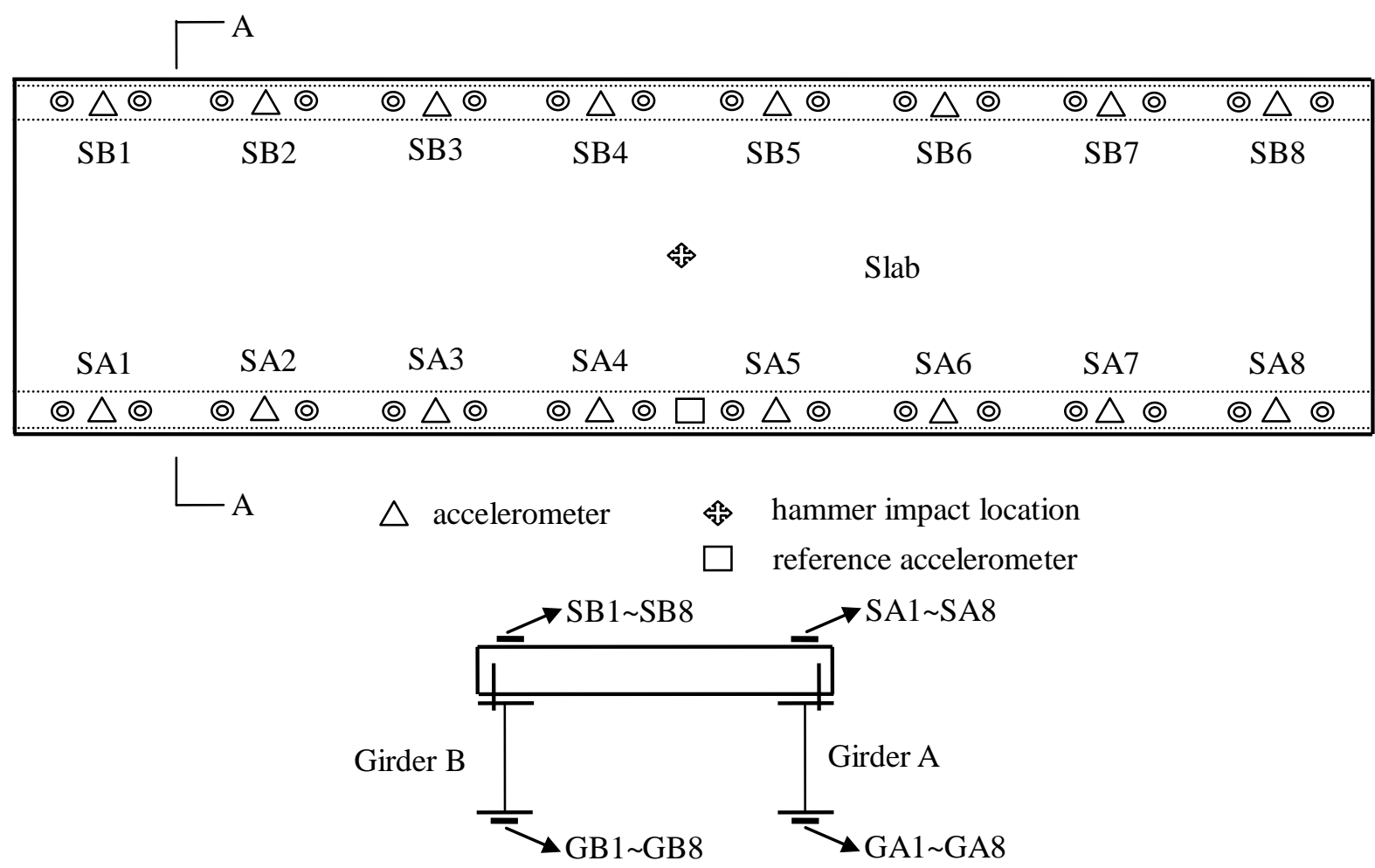

Cross section A - A

Figure 2 - Sensor locations on the slab and underneath the girders 
(a) Damage Scenario 1

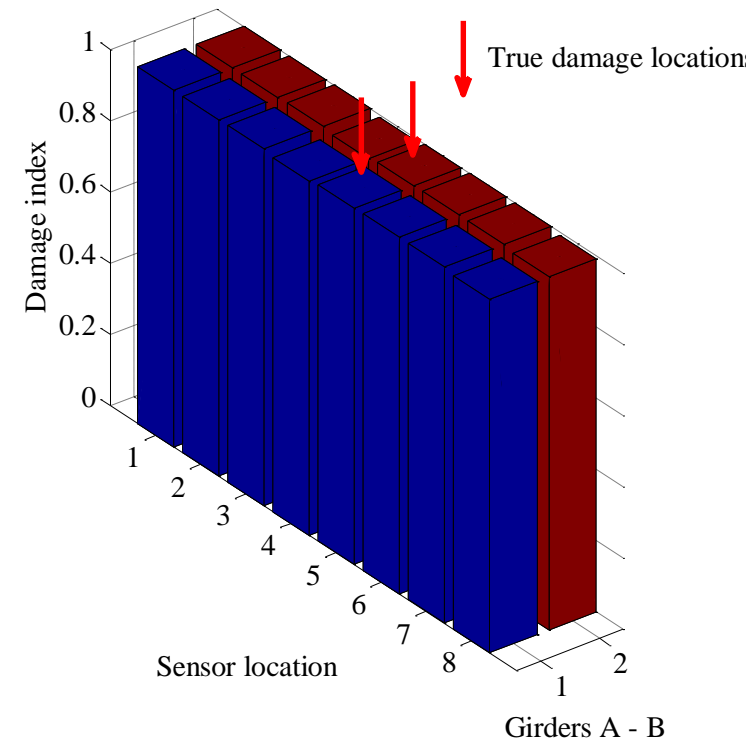

(b) Damage Scenario 2

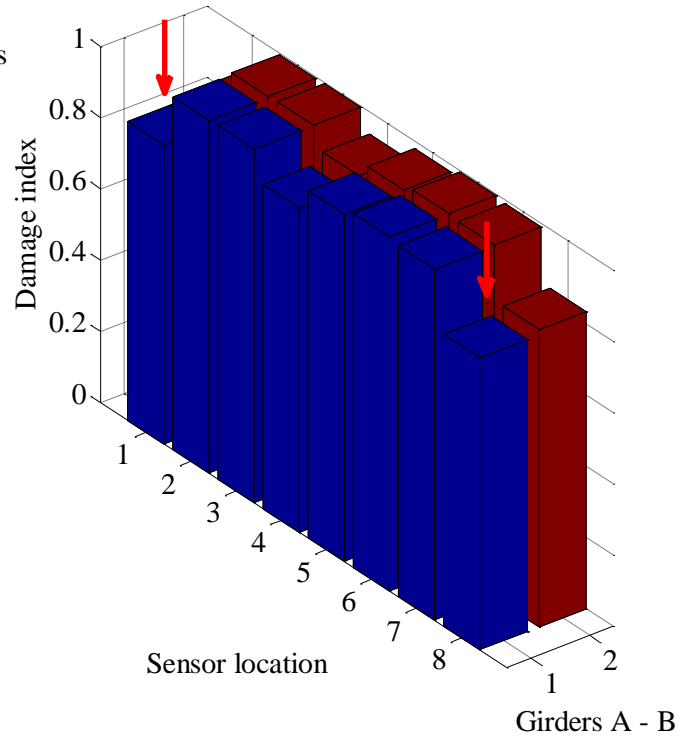

Figure 3 - Damage detection results with COMAC 


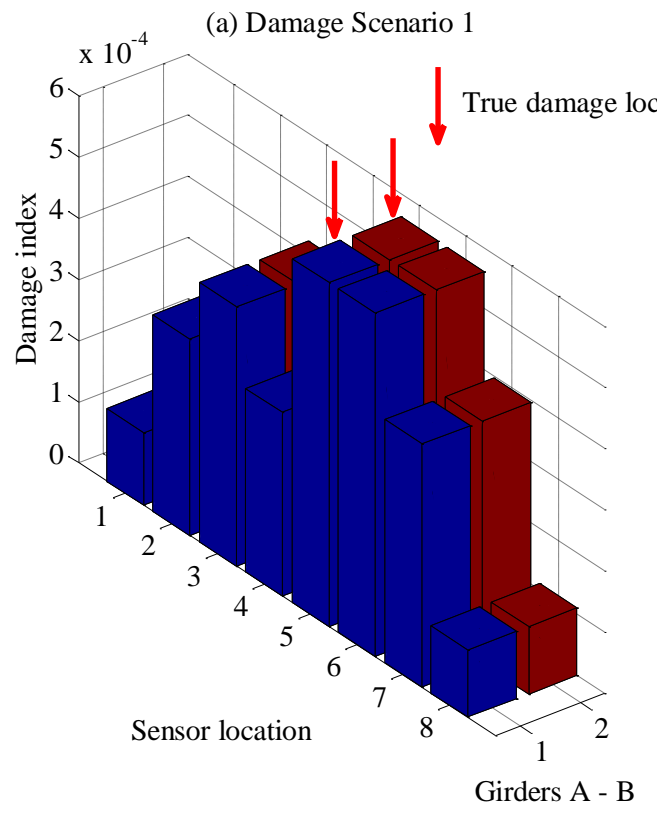

(b) Damage Scenario 2

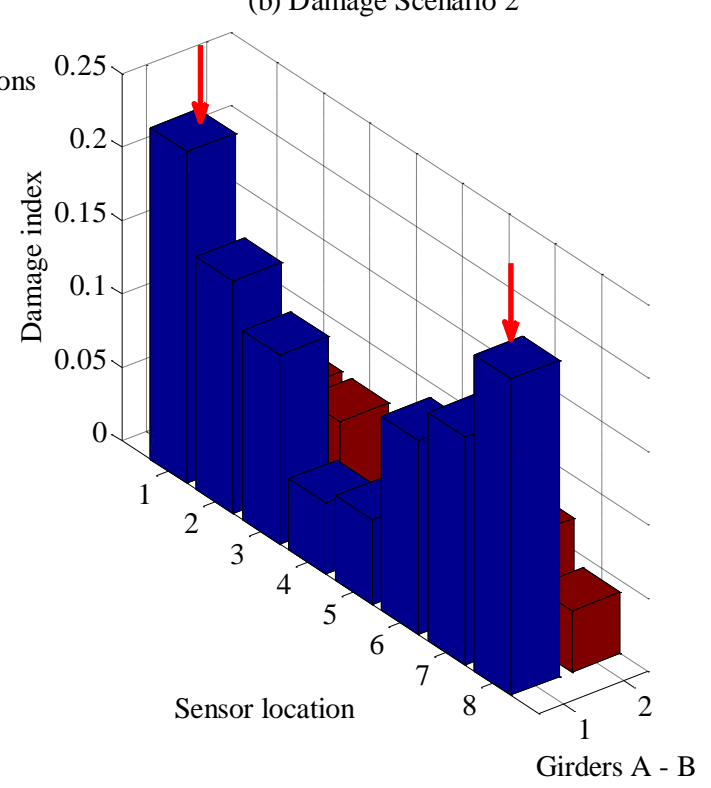

Figure 4 - Damage detection results with changes in flexibility 
(a) Damage Scenario 1

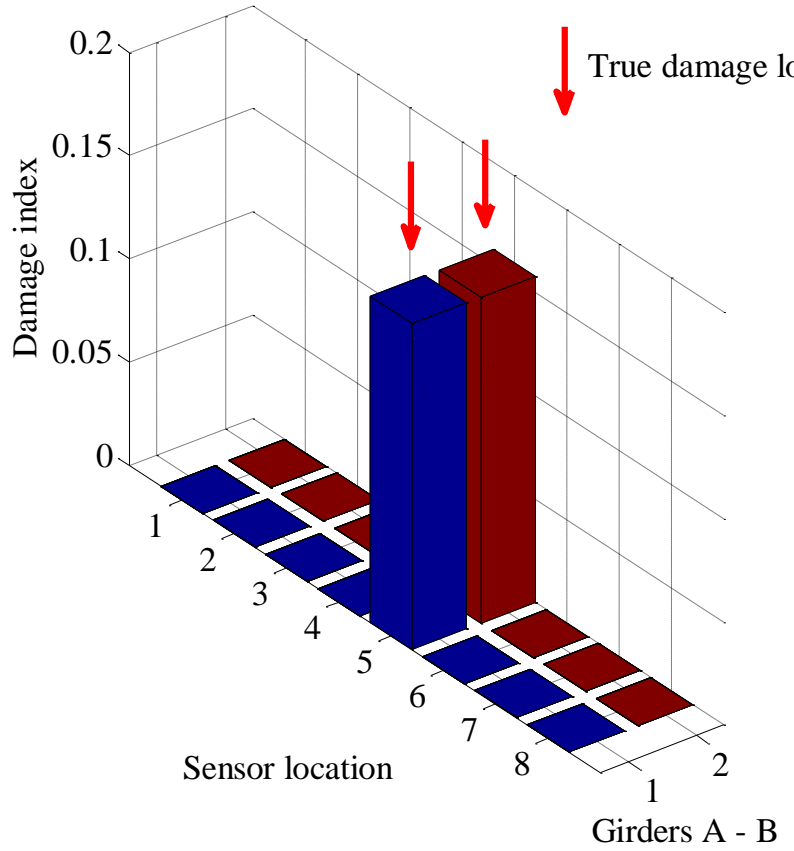

(b) Damage Scenario 2

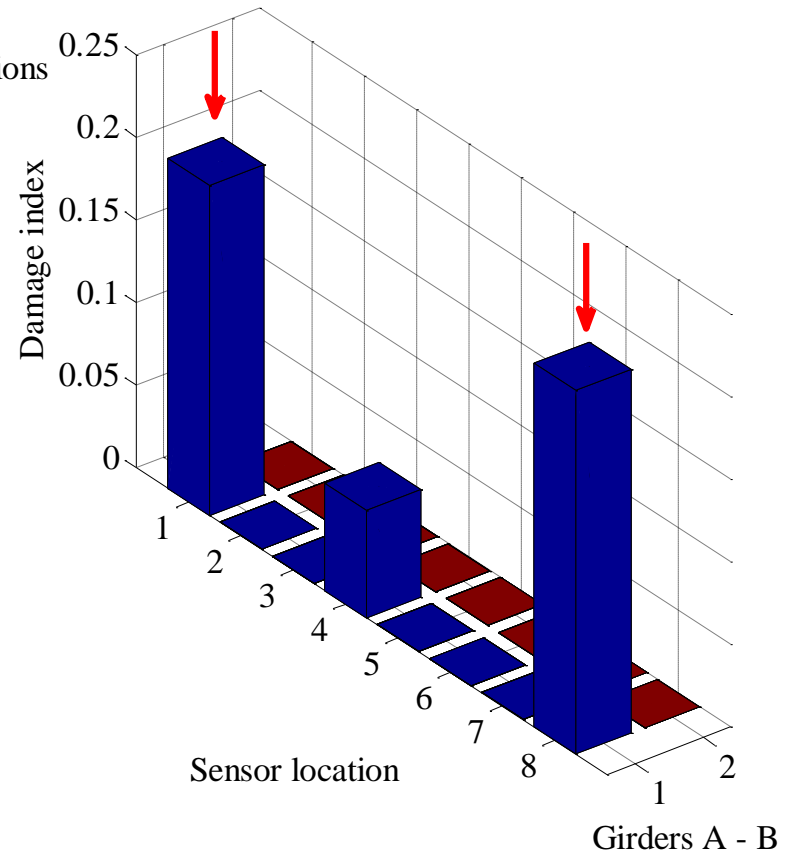

Figure 5 - Damage detection results with measured data of undamaged structure (without noise) 
(1) $3 \%$ noise

(a) Damage Scenario 1

(b) Damage Scenario 2
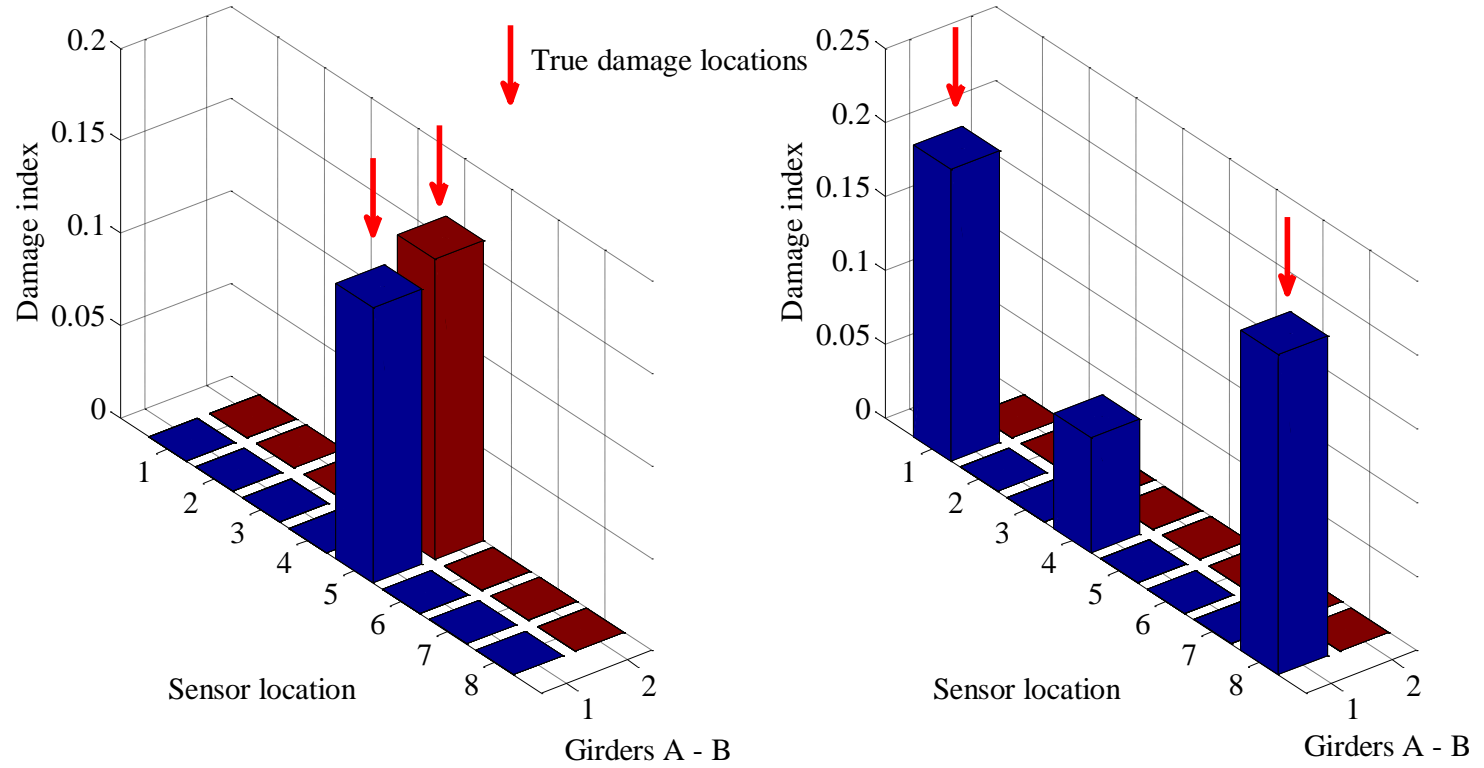

(2) $5 \%$ noise

(a) Damage Scenario 1

(b) Damage Scenario 2
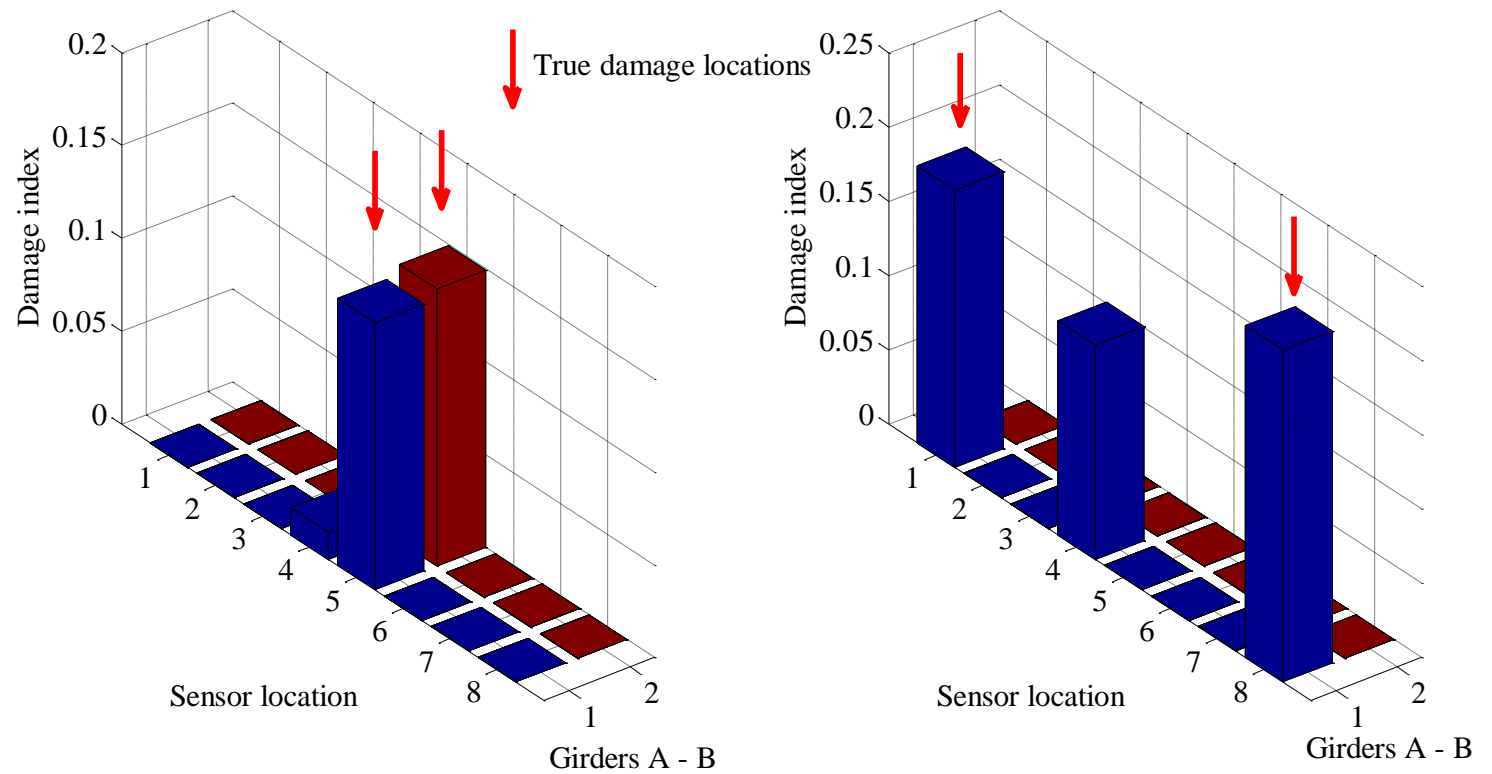

Figure 6 - Damage detection results with measured data of undamaged structure (with noise) 
(a) Damage Scenario 1

(b) Damage Scenario 2
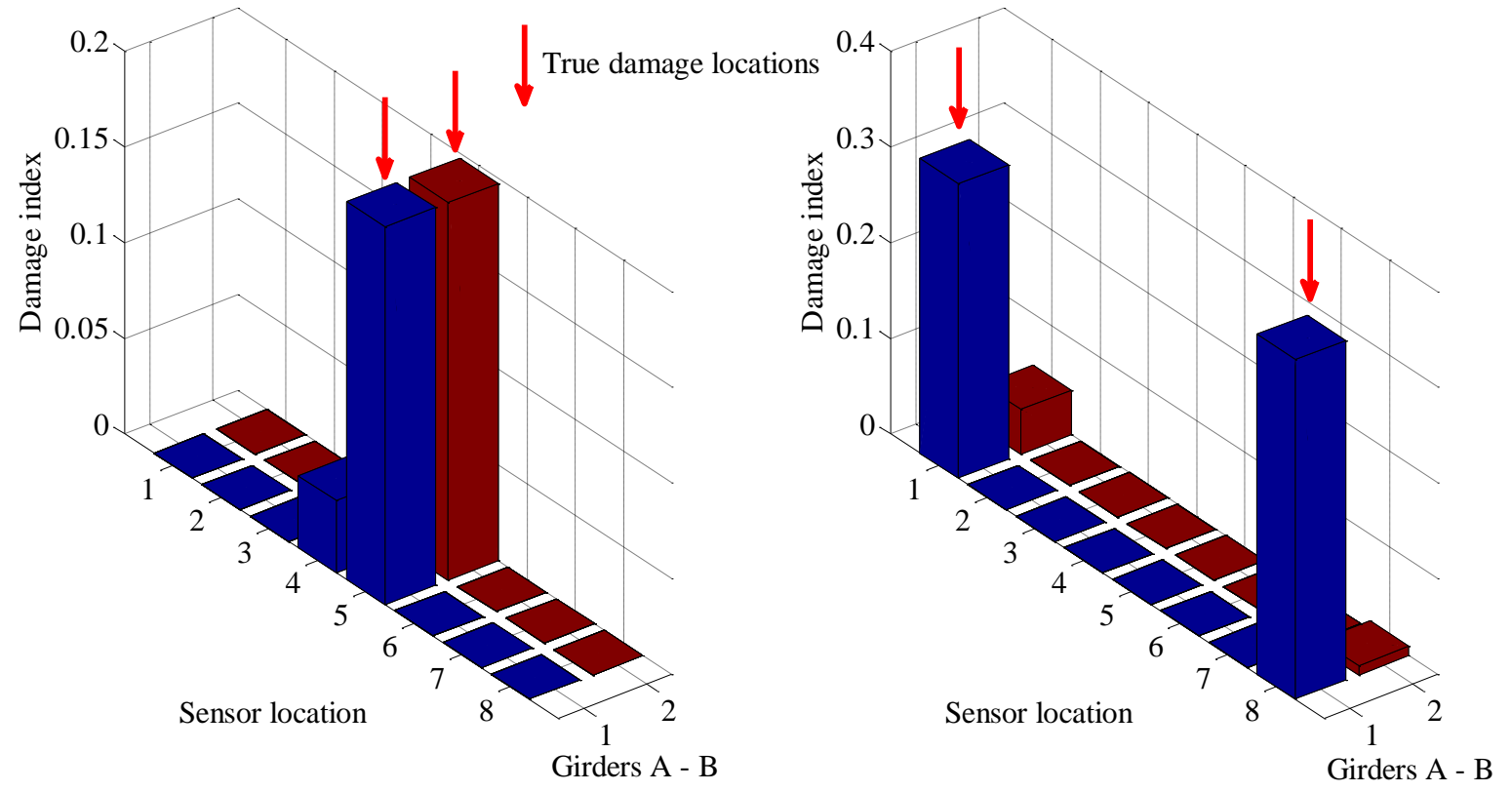

Figure 7 - Damage detection results with RDFRF (without noise) 
(1) $3 \%$ noise

(a) Damage Scenario 1

(b) Damage Scenario 2
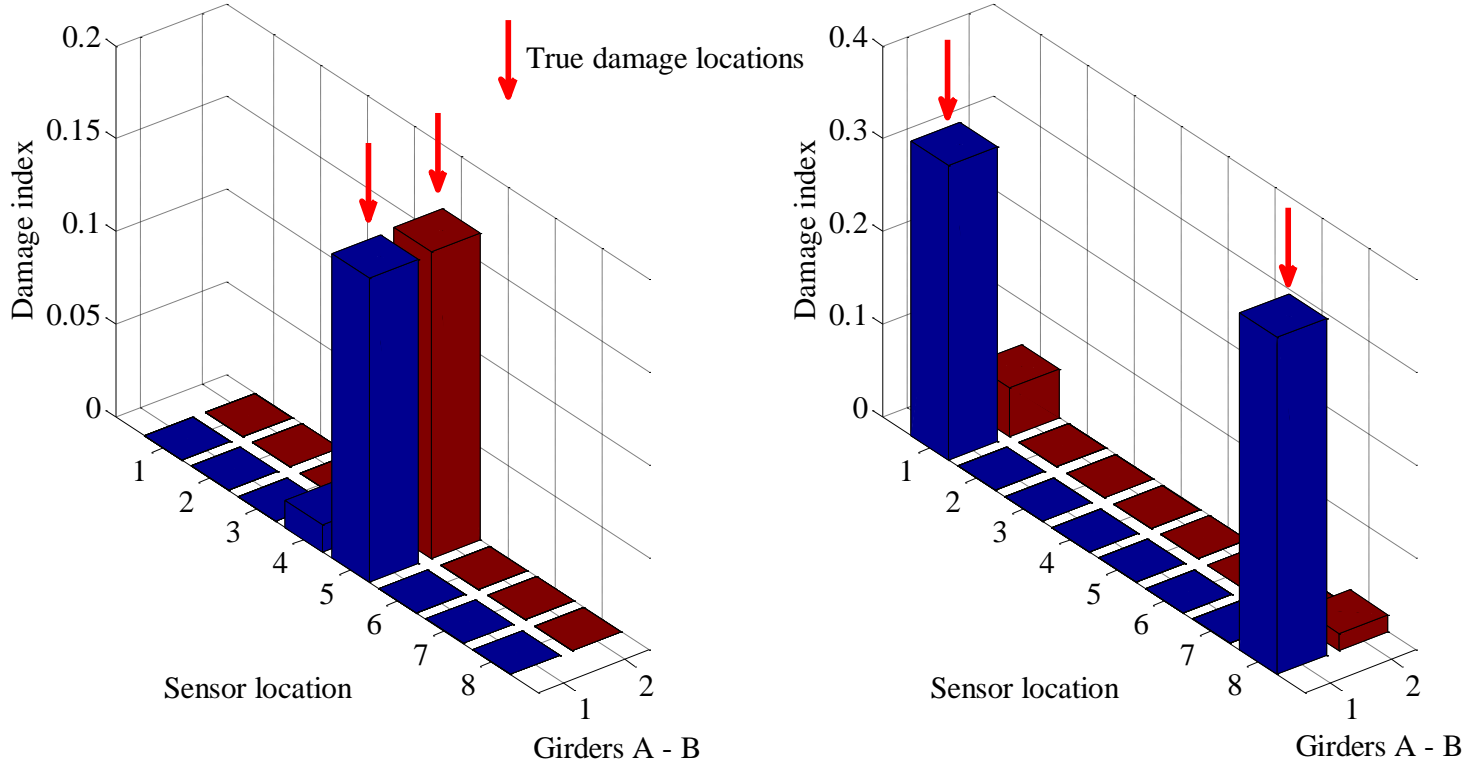

(2) $5 \%$ noise

(a) Damage Scenario 1

(b) Damage Scenario 2
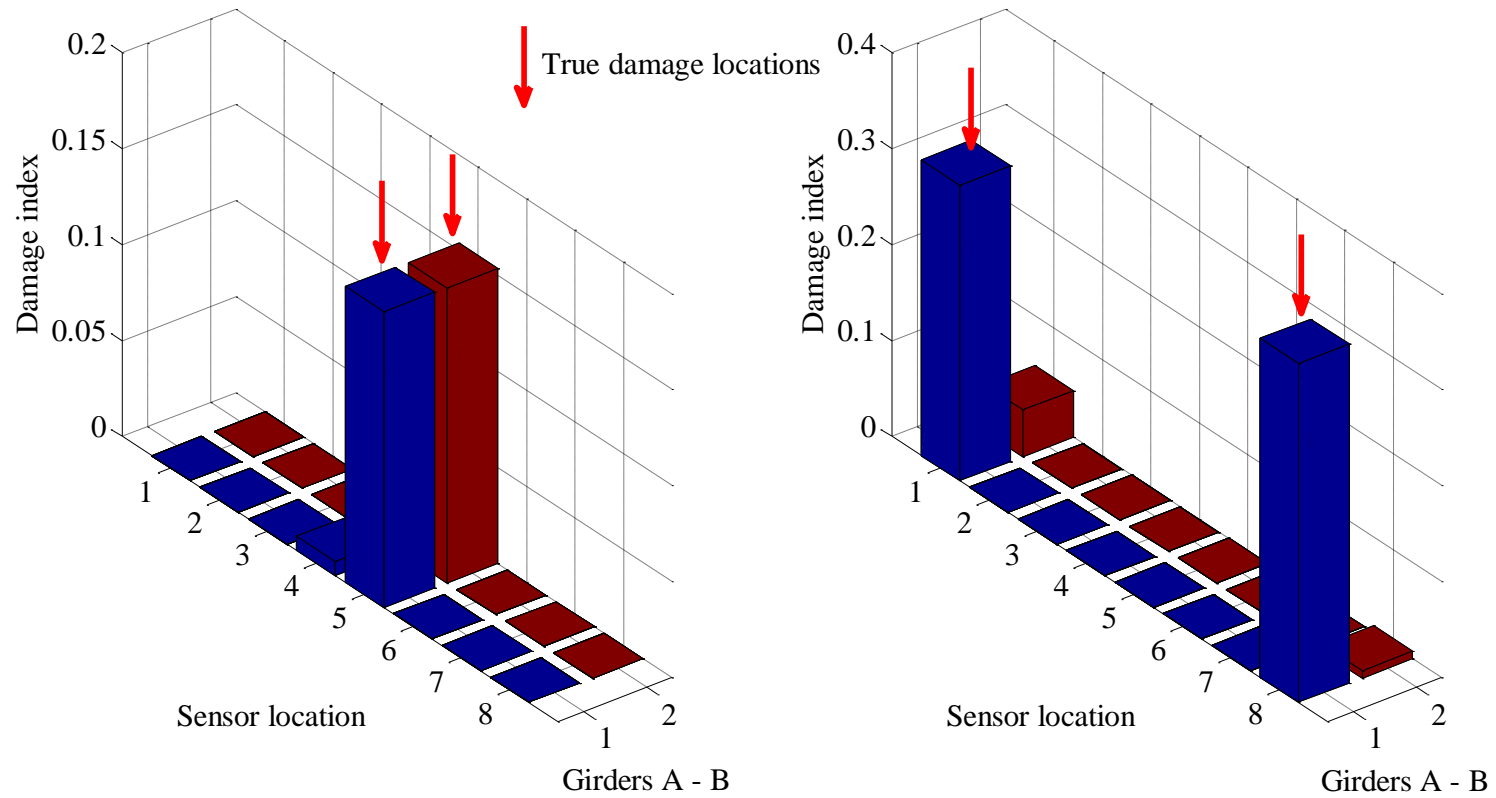

Figure 8 - Damage detection results with RDFRF (with noise) 
(a) Damage Scenario 1

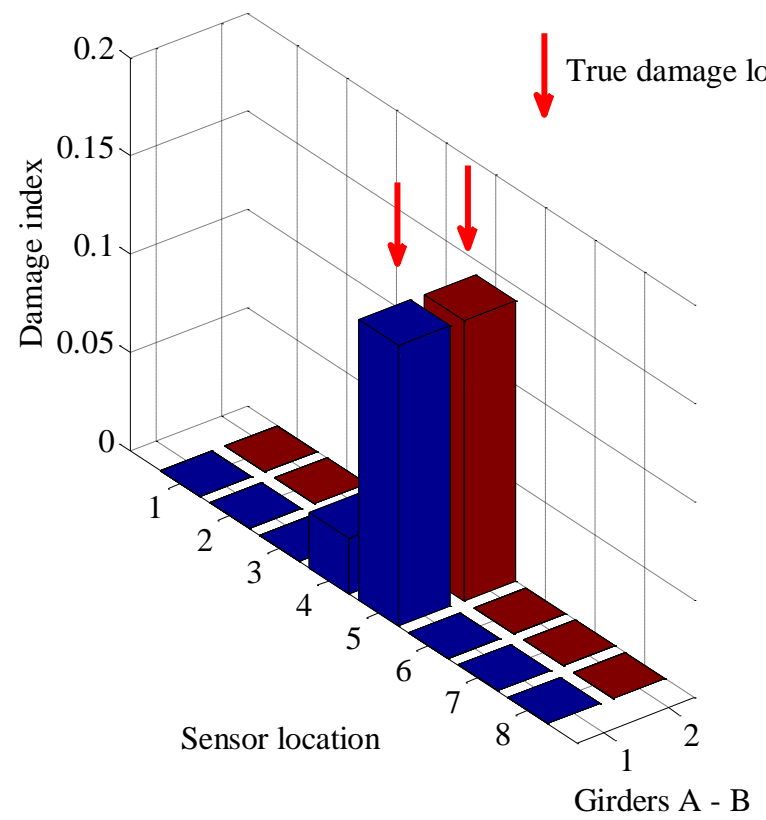

(b) Damage Scenario 2

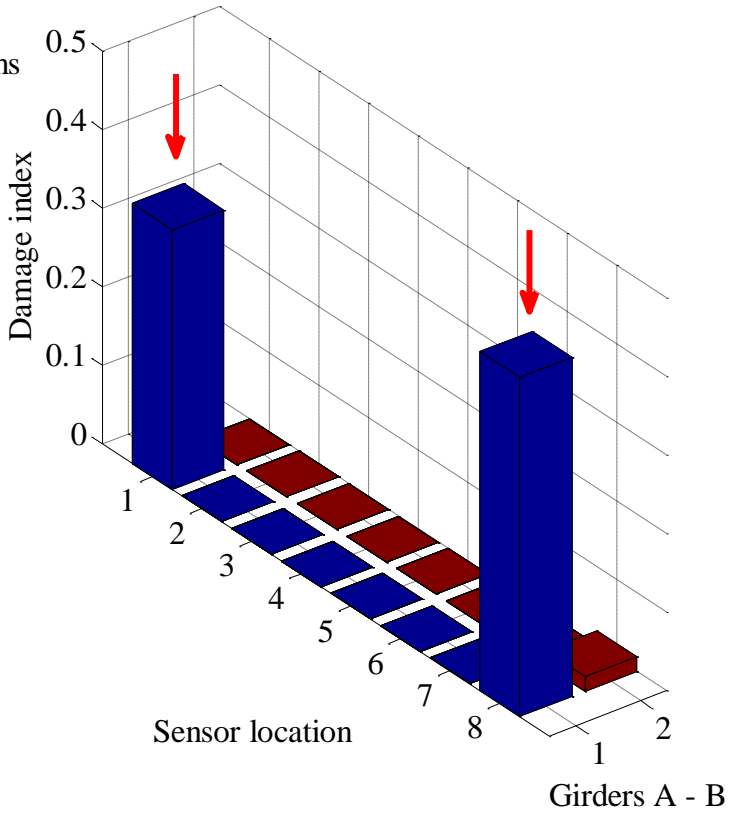

Figure 9 - Damage detection results without noise and data of undamaged structure 
(1) $3 \%$ noise

(a) Damage Scenario 1

(b) Damage Scenario 2
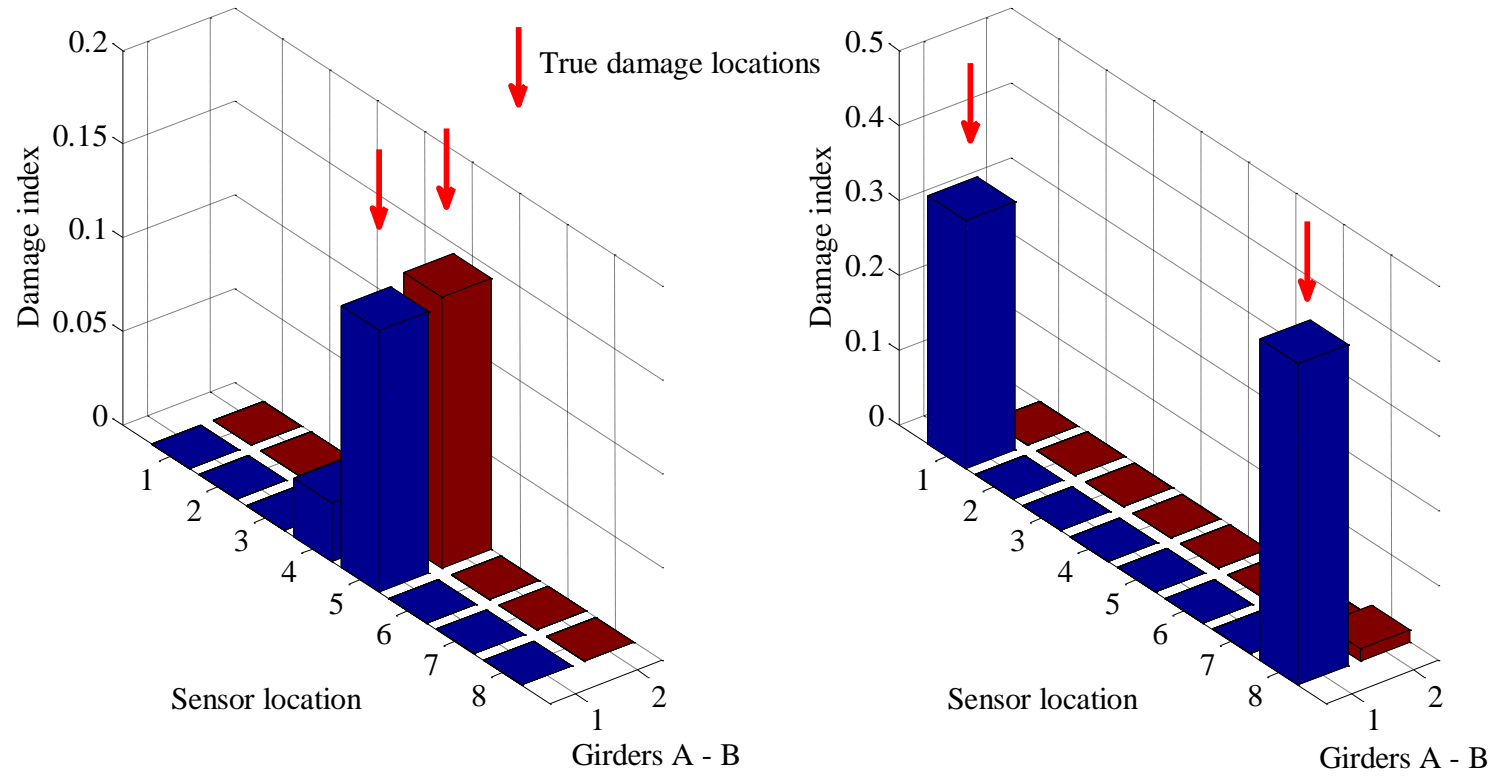

(2) $5 \%$ noise

(a) Damage Scenario 1

(b) Damage Scenario 2
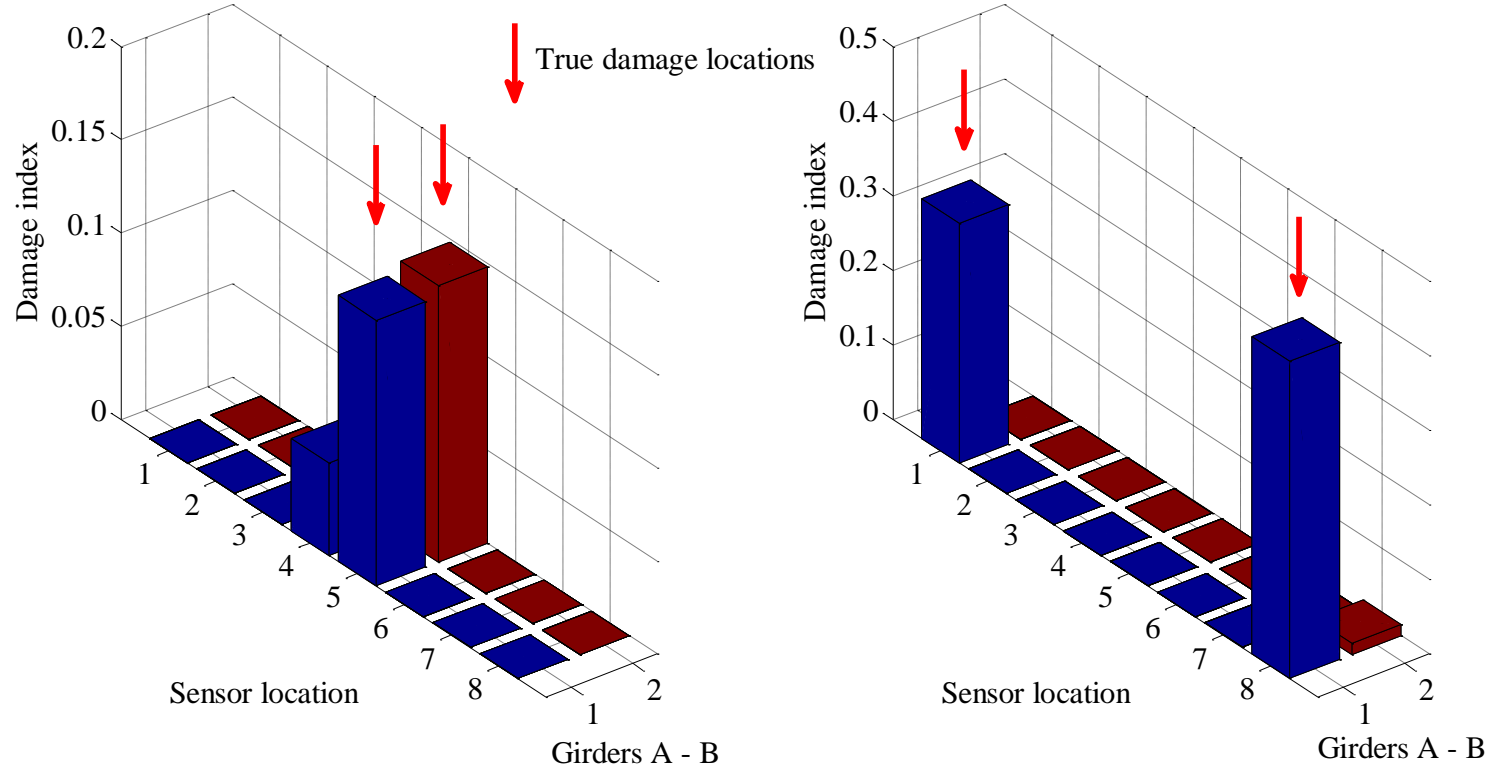

Figure 10 - Damage detection results with noise and without data of undamaged structure 


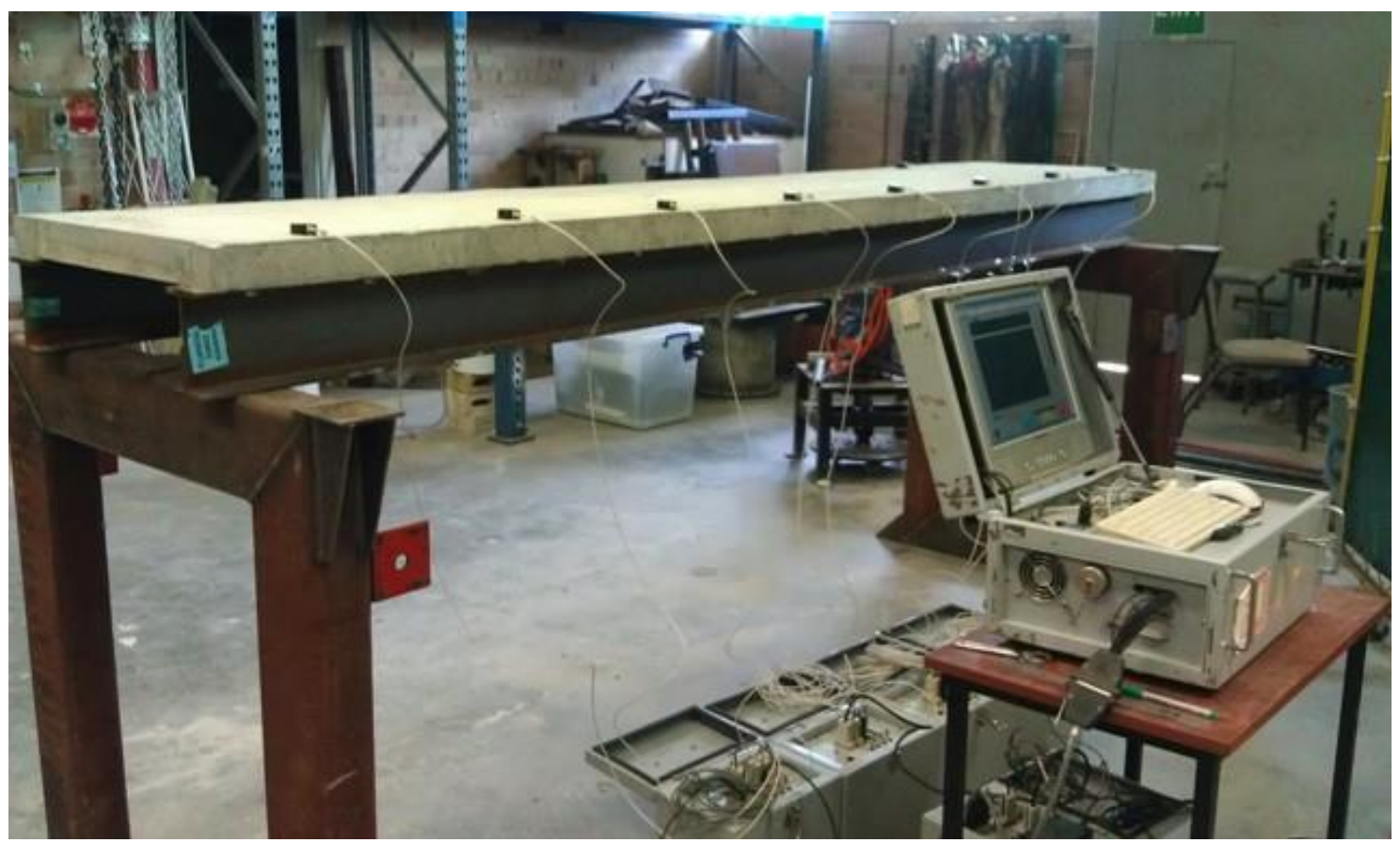

Figure 11 - Experimental test setup of the slab-on-girder structure 


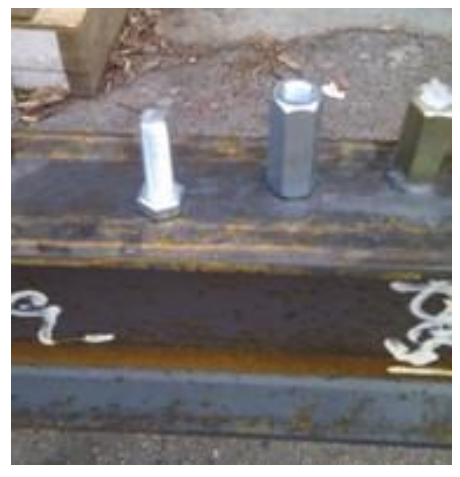

(a) Metric bolt and nut

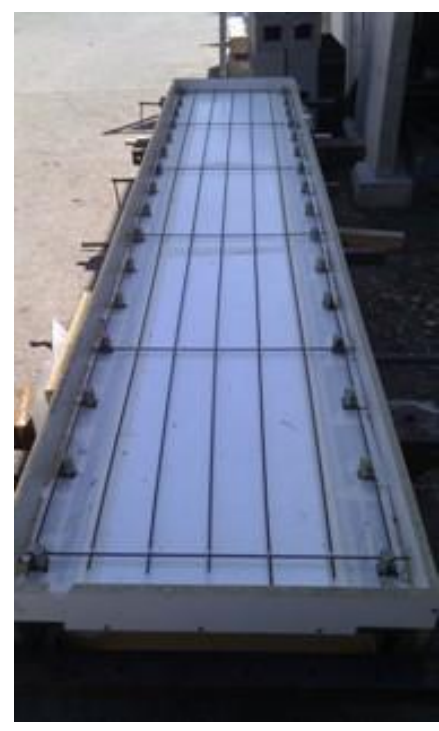

(c) Plan view of shear connectors

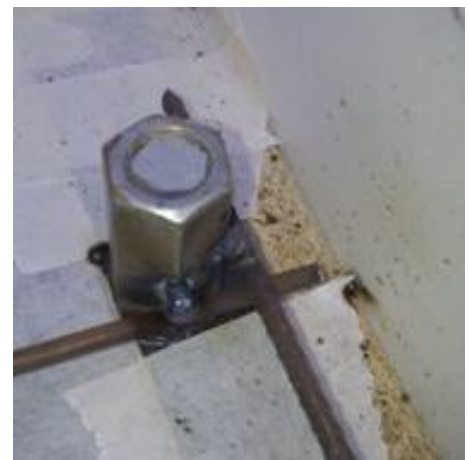

(b) Bolt screwed into the nut

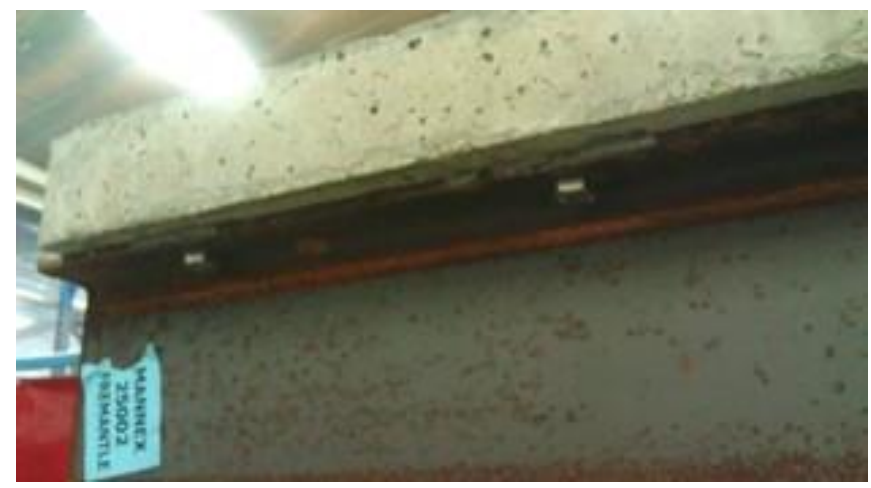

(d) Shear connector in the structure

Figure 12 - Design of shear connectors 


\begin{tabular}{|c|c|c|c|c|c|c|c|}
\hline SC17 SC18 & SC19 SC2 & $\mathrm{SC} 21 \mathrm{SC} 2$ & SC23 SC2 & 4 SC25 SC26 & $\mathrm{SC} 27 \mathrm{SC} 28$ & SC29 SC30 & $\mathrm{SC} 31 \mathrm{SC} 32$ \\
\hline @ $\triangle \Theta$ & $\odot \triangle \odot$ & $\odot \triangle \odot$ & Q $\triangle \triangle 0$ & $@ \triangle @$ & $@ \triangle @$ & $\odot \triangle \odot$ & @ $\triangle 0$ \\
\hline \multirow[t]{2}{*}{ SB1 } & SB2 & SB3 & SB4 & SB5 & SB6 & SB7 & SB8 \\
\hline & \multicolumn{2}{|c|}{ 雪 } & \multicolumn{5}{|c|}{ Slab } \\
\hline SA1 & SA2 & SA3 & SA4 & SA5 & SA6 & SA7 & SA8 \\
\hline$\odot \triangle \odot$ & ( $\triangle \odot$ & $\odot \triangle \odot$ & ๑ $\triangle \odot$ & $\square \odot \triangle \odot$ & $(\triangle \bigcirc$ & $\odot \triangle \odot$ & $(\triangle \triangle \odot$ \\
\hline
\end{tabular}

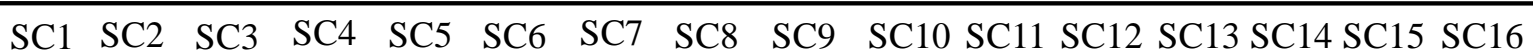

LA $\triangle$ shear connector $\triangle$ accelerometer hammer impact location

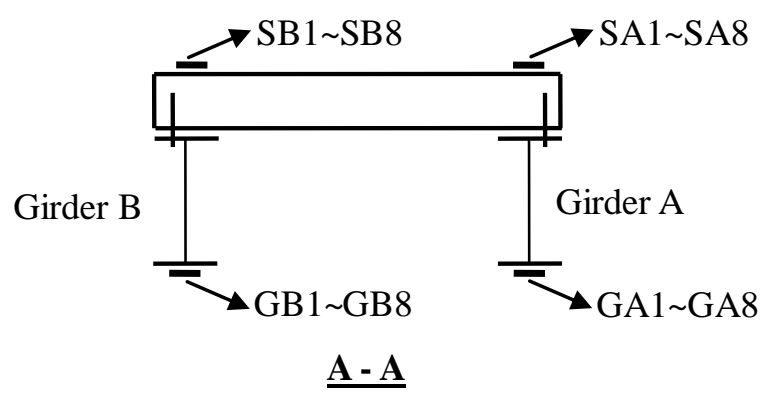

Figure 13- Experimental sensor placement 
(1) First mode

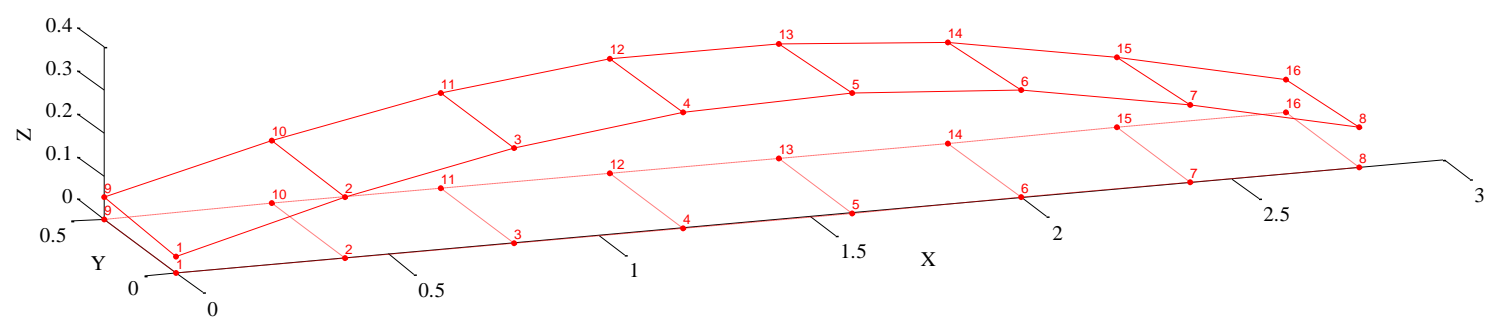

(2) Second mode

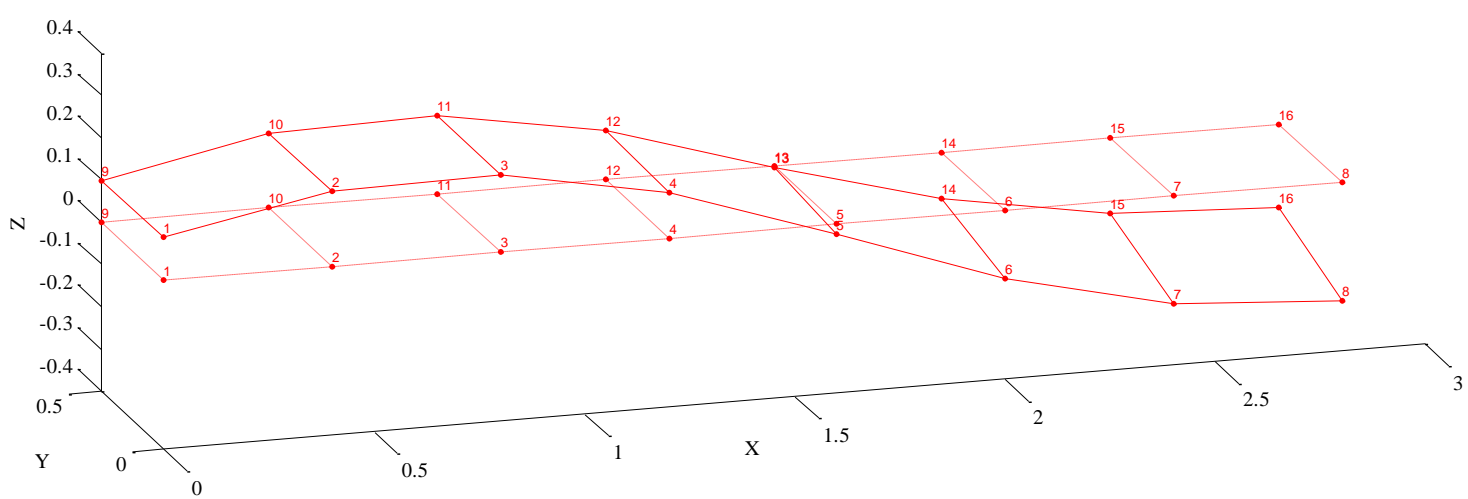

(3) Third mode

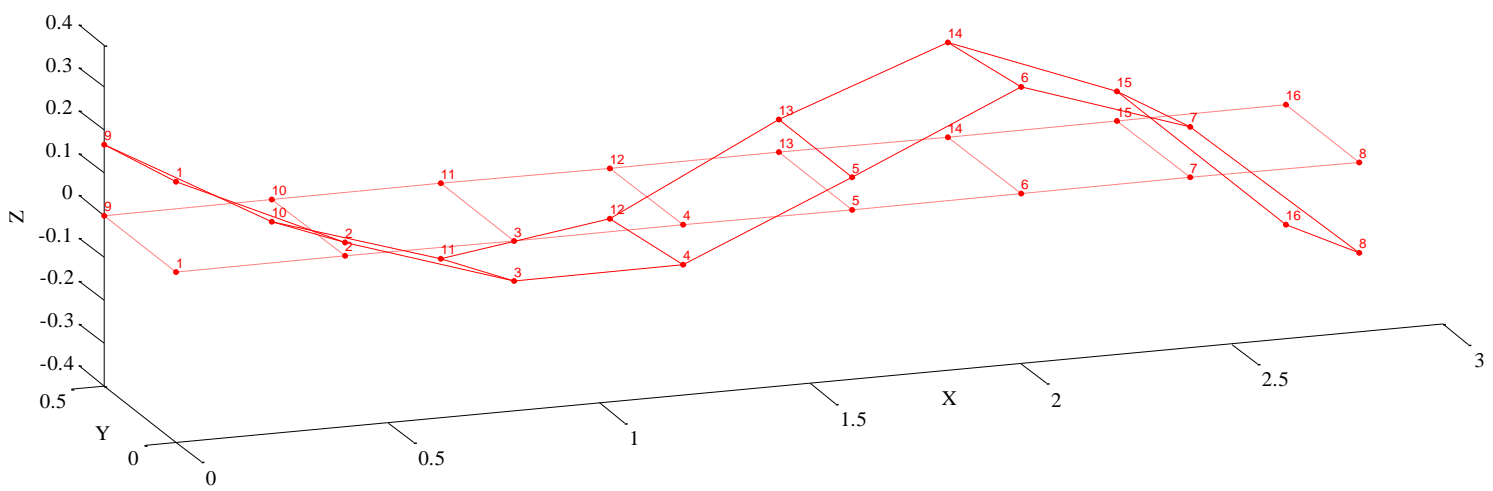

Figure 14 - Identified first three mode shapes of the undamaged structure 
(a) Damage Scenario 1

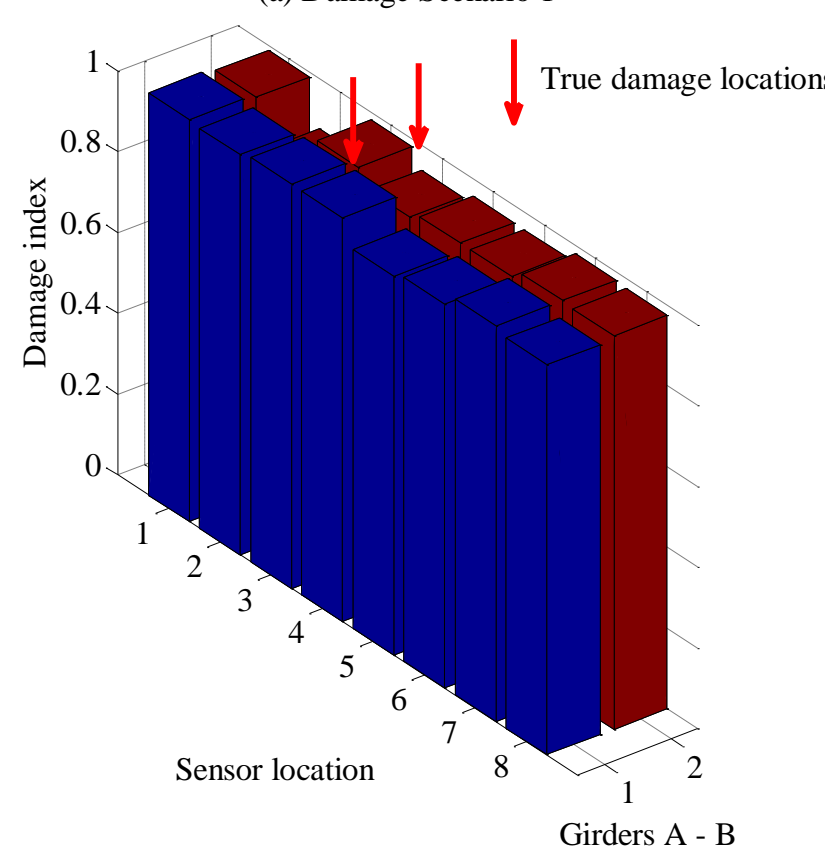

(b) Damage Scenario 2

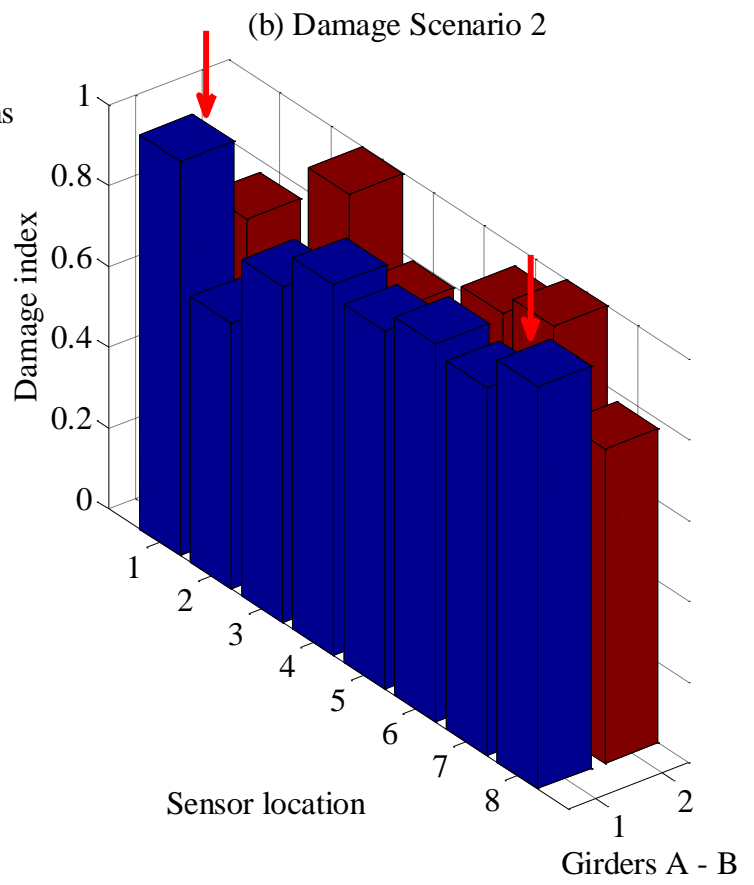

Figure 15 - Damage detection results with COMAC 

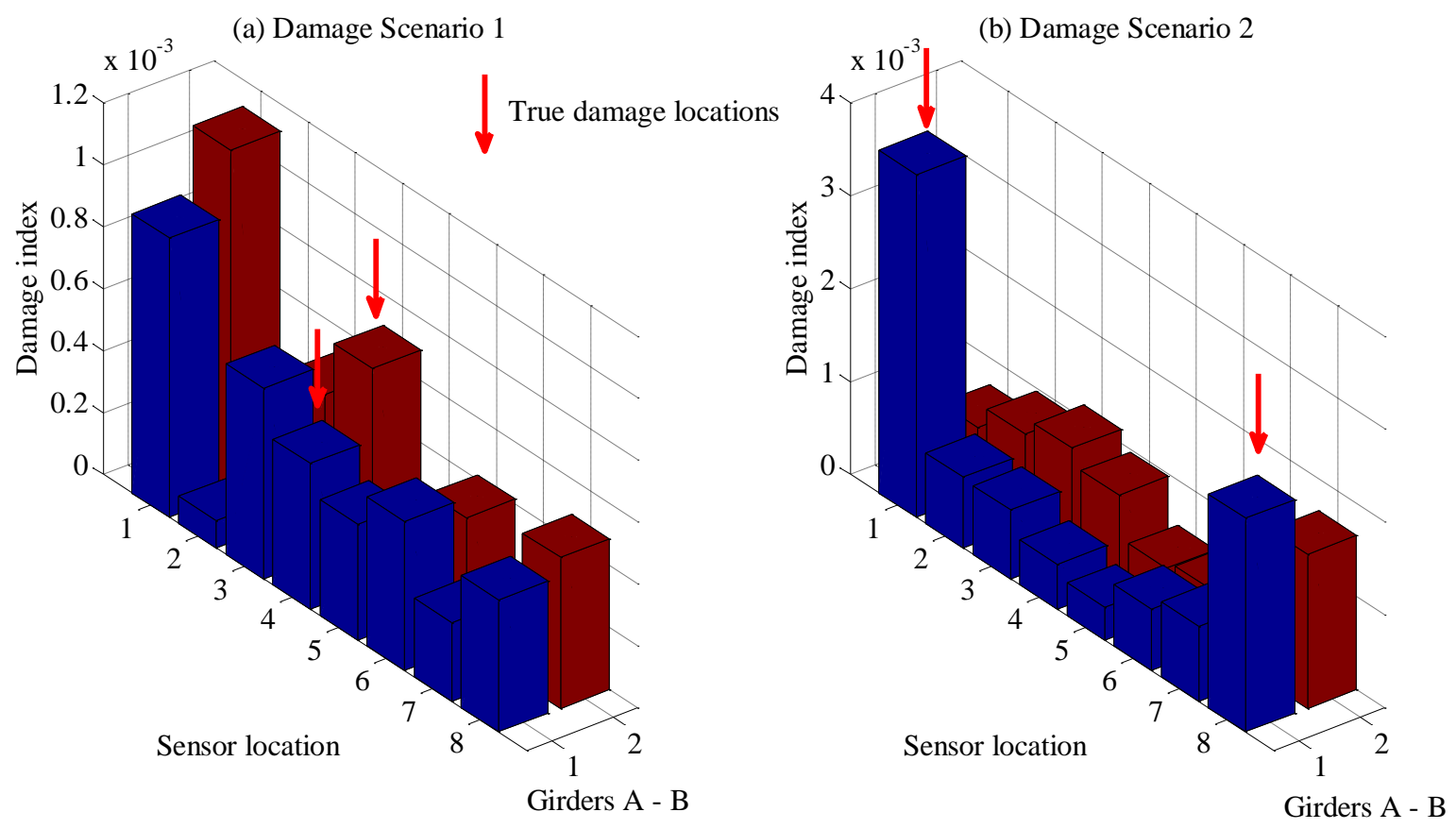

Figure 16 - Damage detection with changes in flexibility 


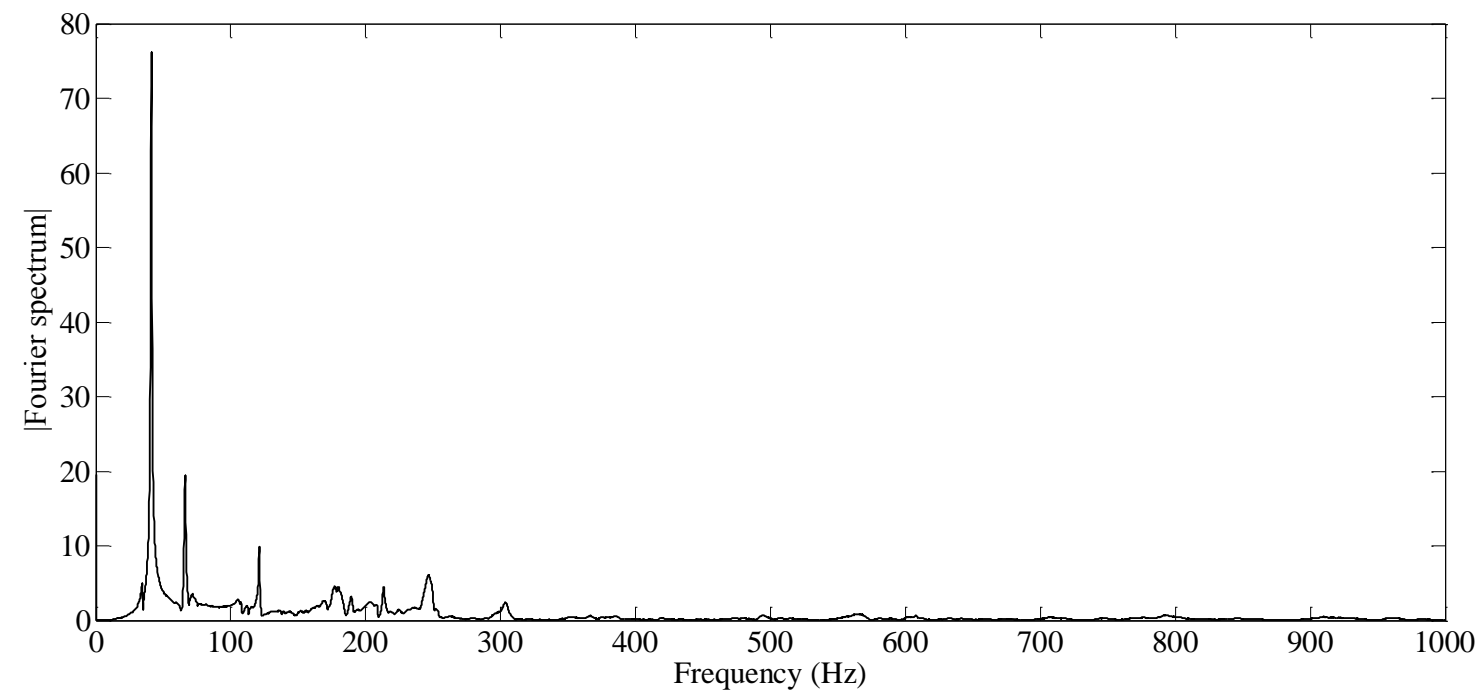

Figure 17 - Fourier spectrum of the measured response at SB5 in the damaged state 
(a) Damage Scenario 1

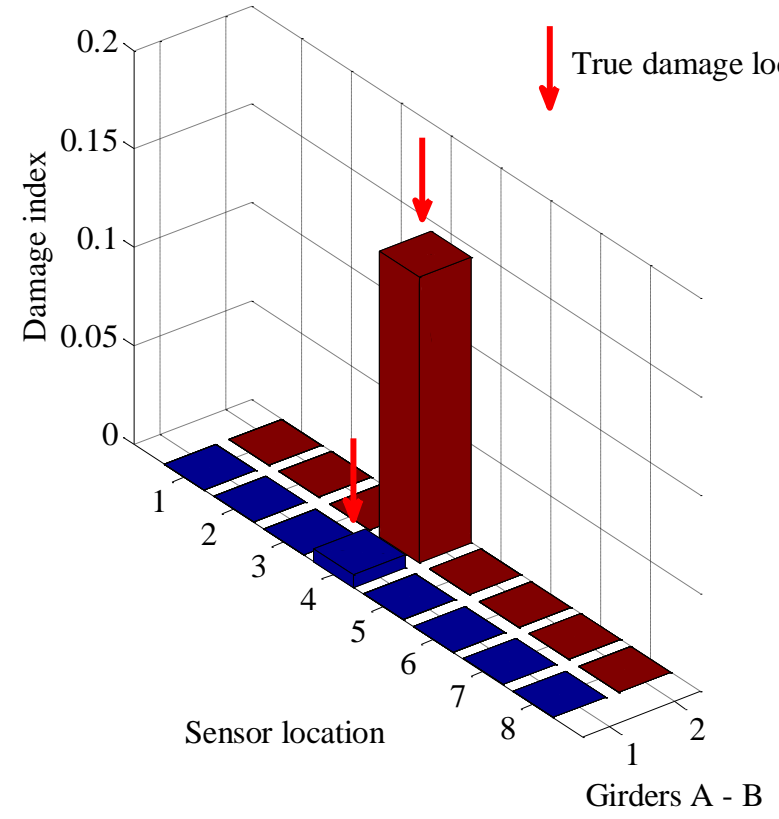

(b) Damage Scenario 2

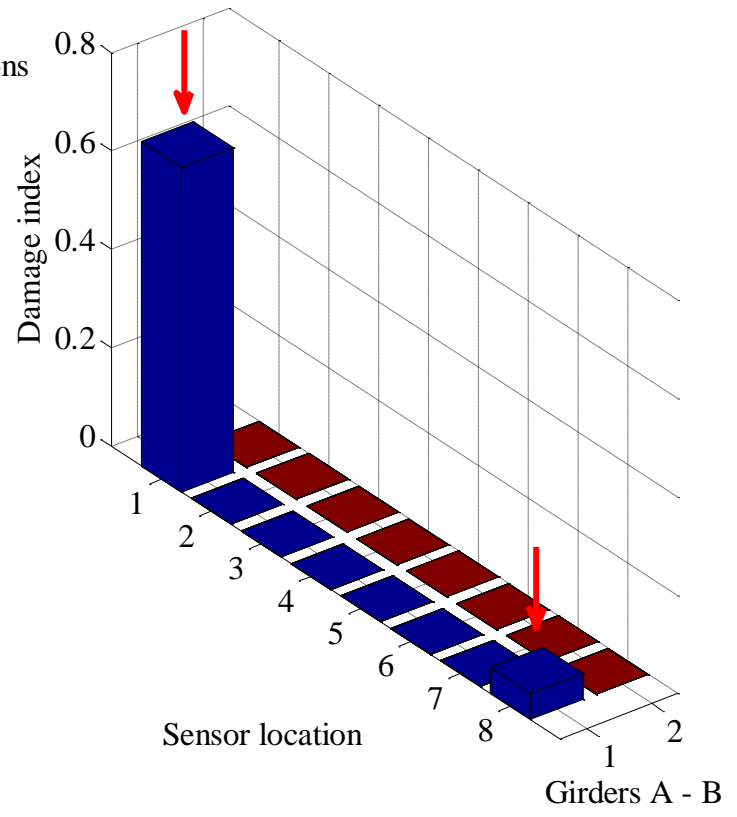

Figure 18 - Damage detection results with measured data of undamaged structure 
(a) Damage Scenario 1

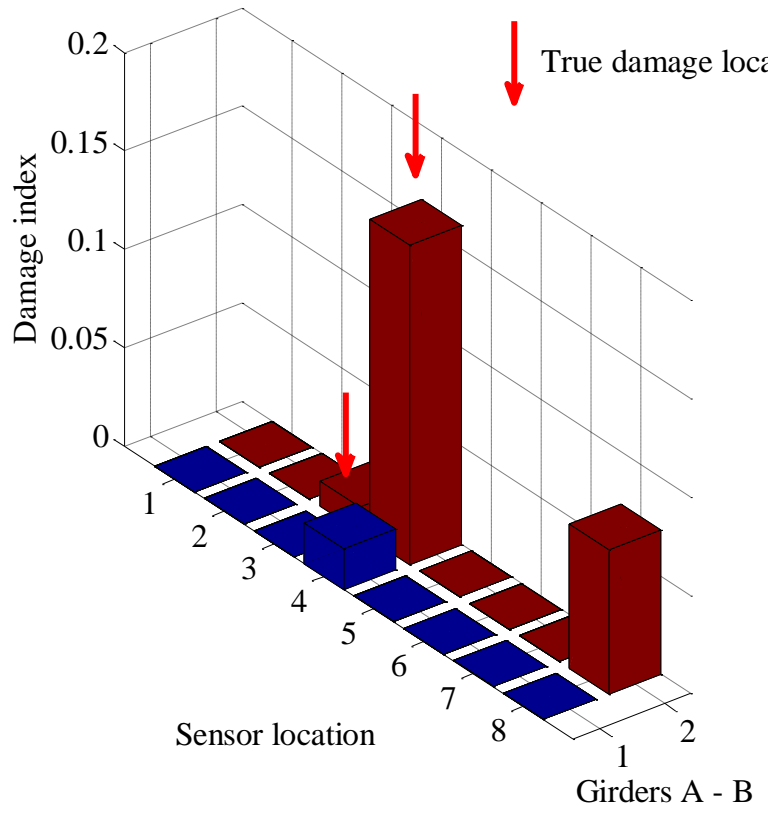

(b) Damage Scenario 2

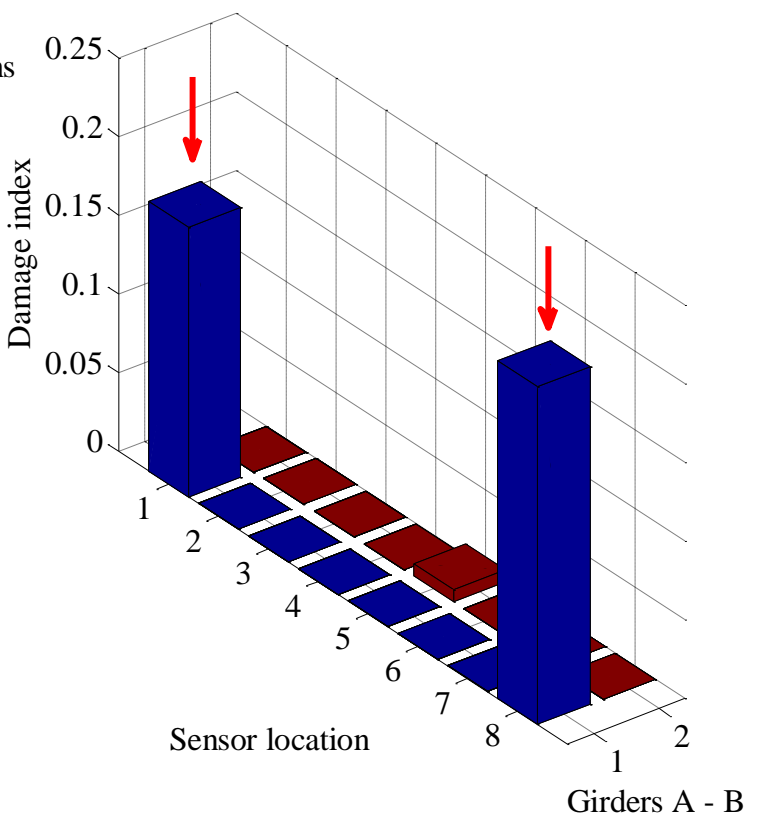

Figure 19 - Damage detection results with RDFRF 


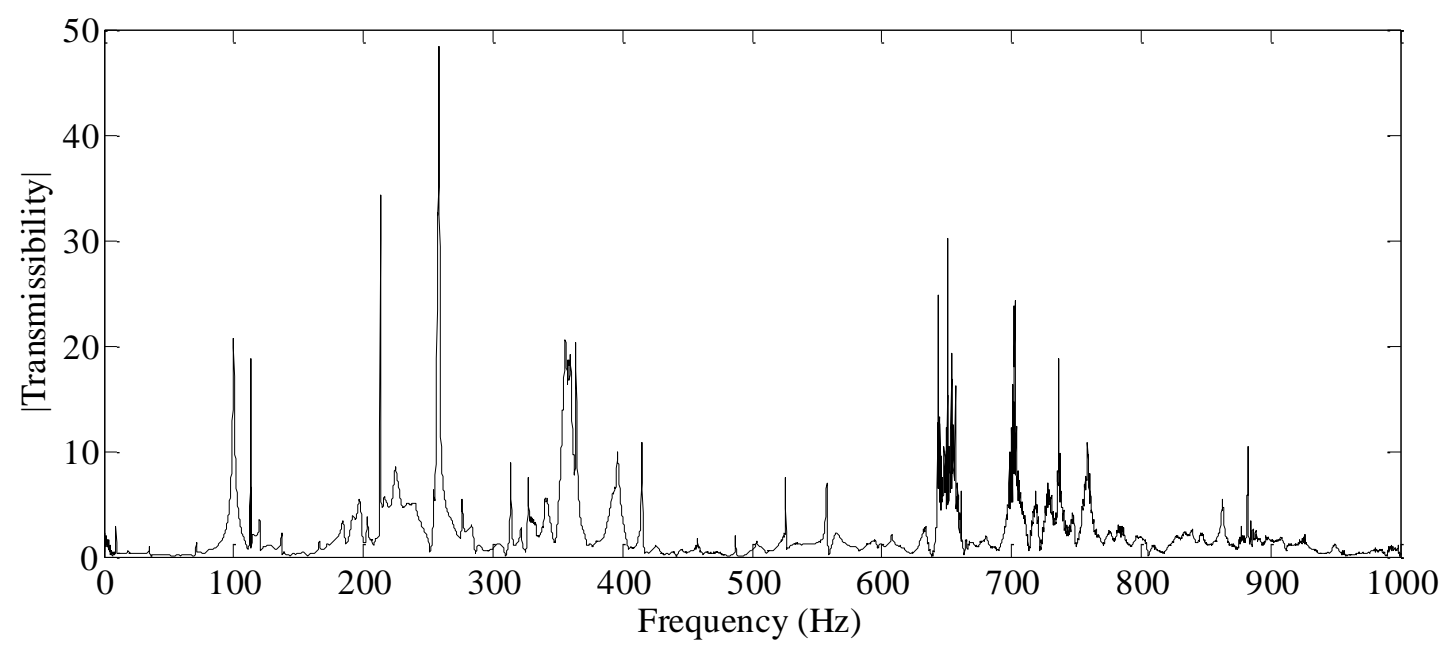

Figure 20 - Transmissibility at sensor location SA1 of damage Scenario 1 
(a) Damage Scenario 1
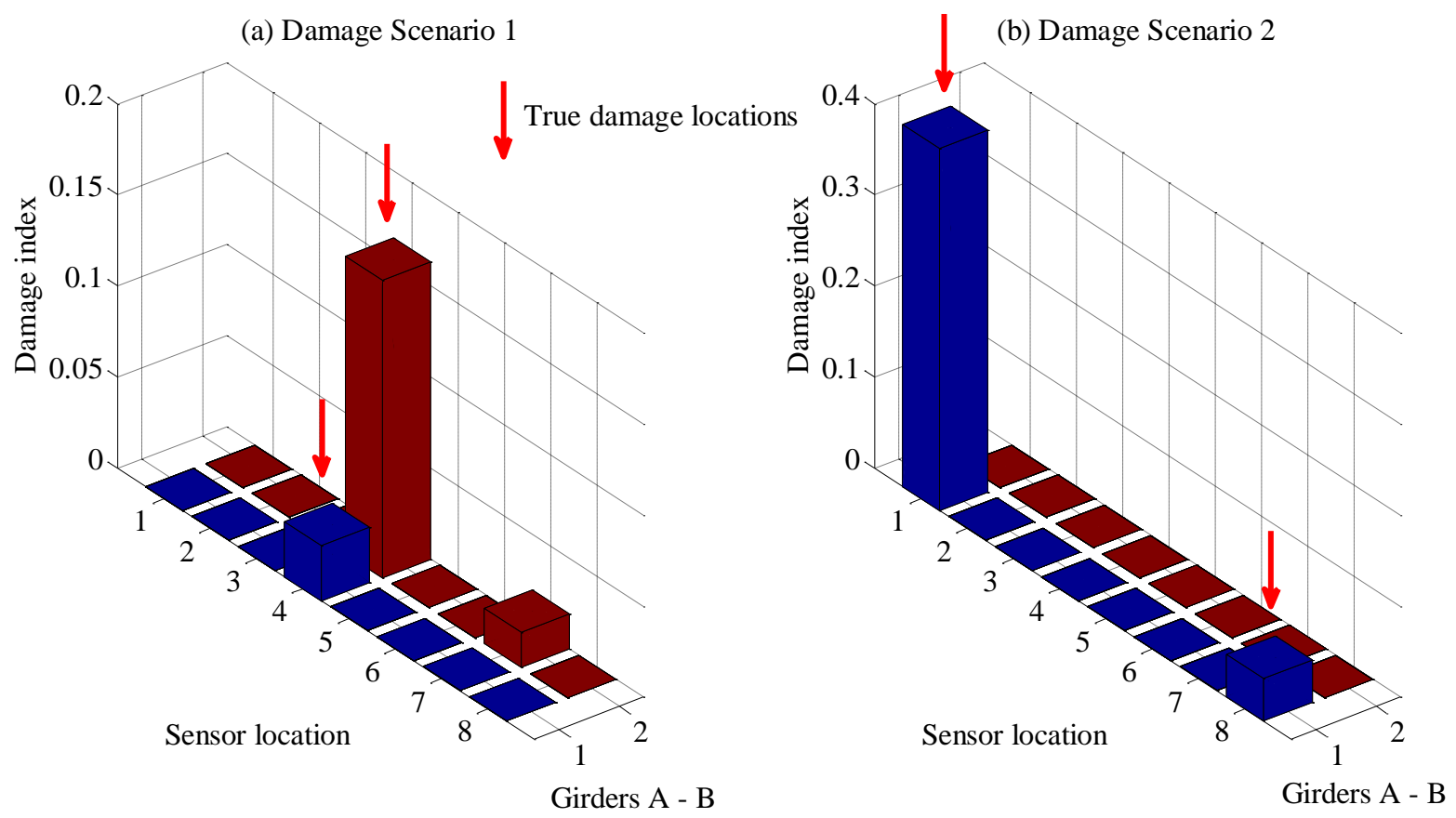

Figure 21 - Damage detection results without measured data of undamaged structure 

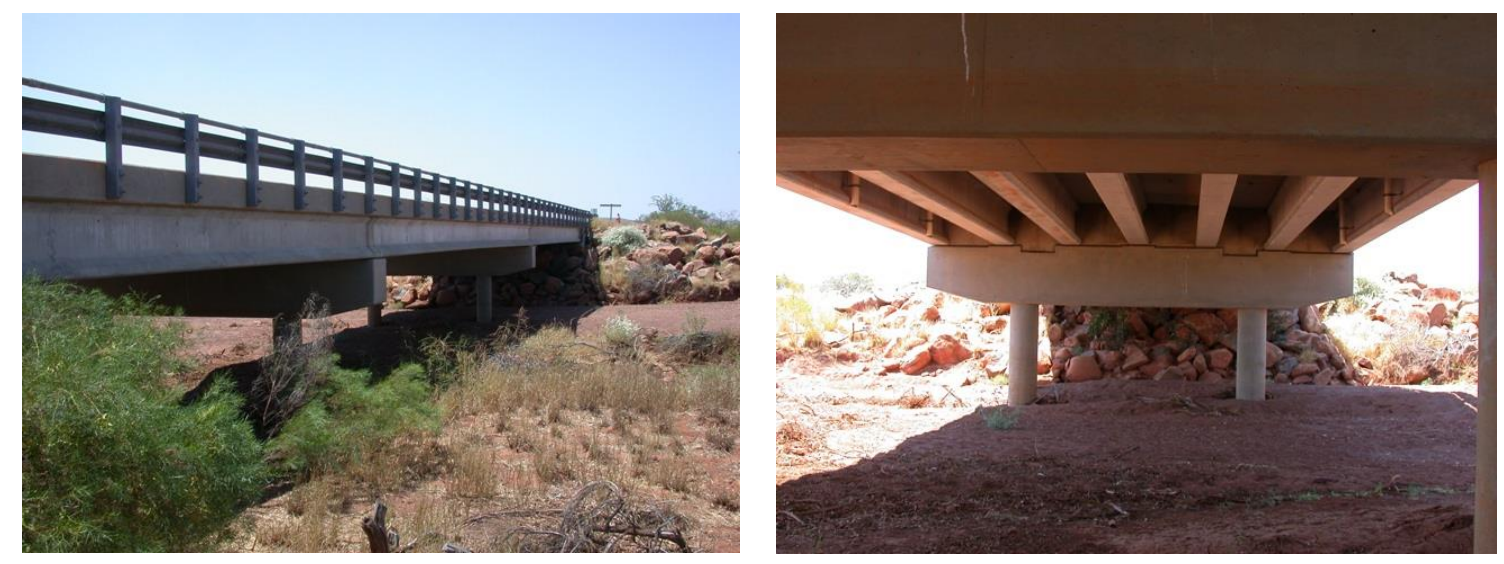

Figure 22 - General view of Bridge No. 852 
(a) Slab

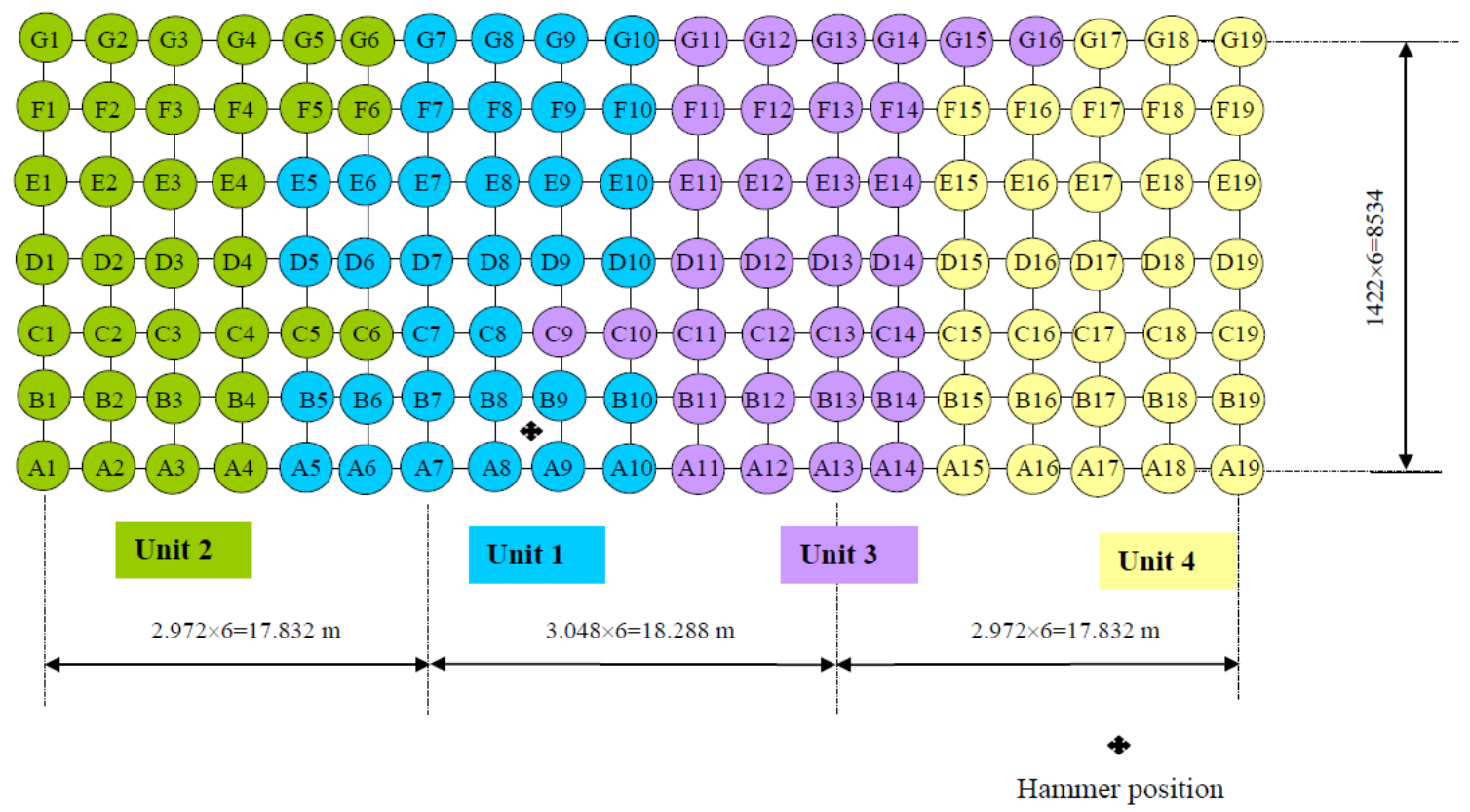

(b) Girders

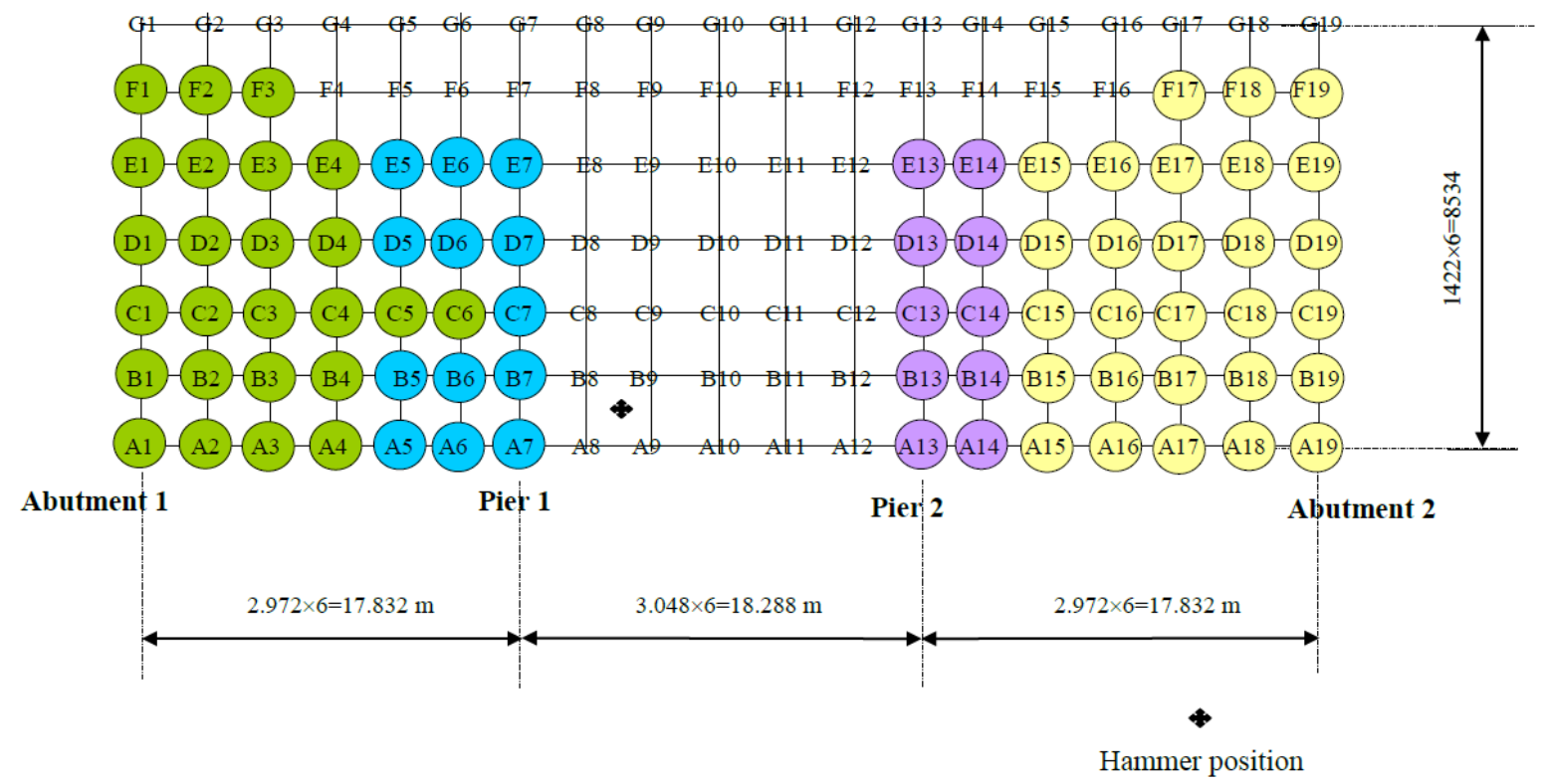

Figure 23 - Sensor placement on slab and girders 
(a)

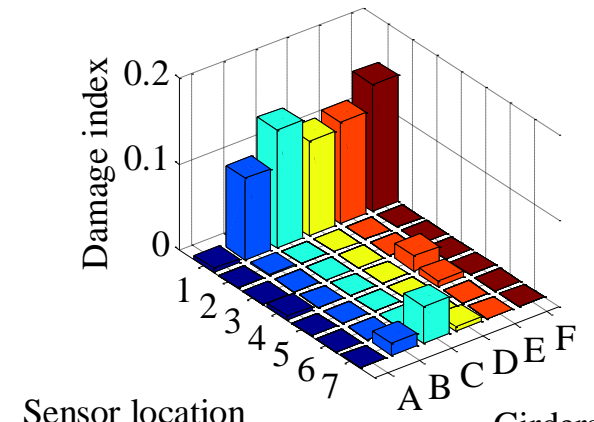

(c)

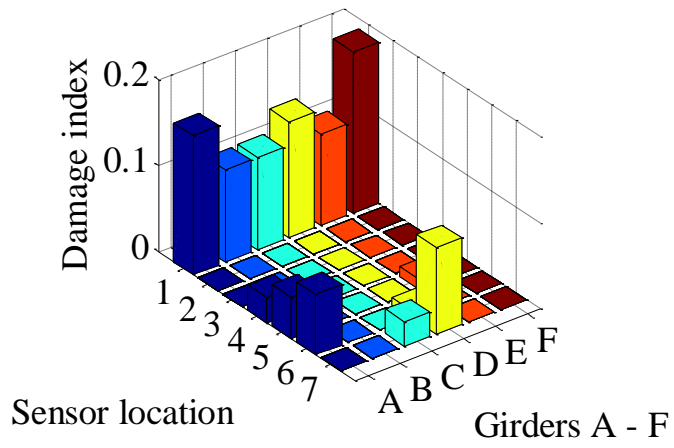

(b)

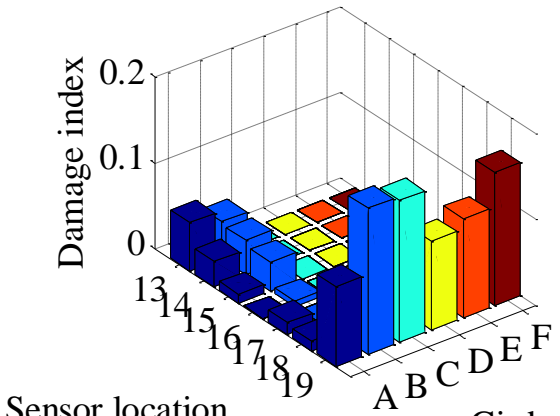

(d)

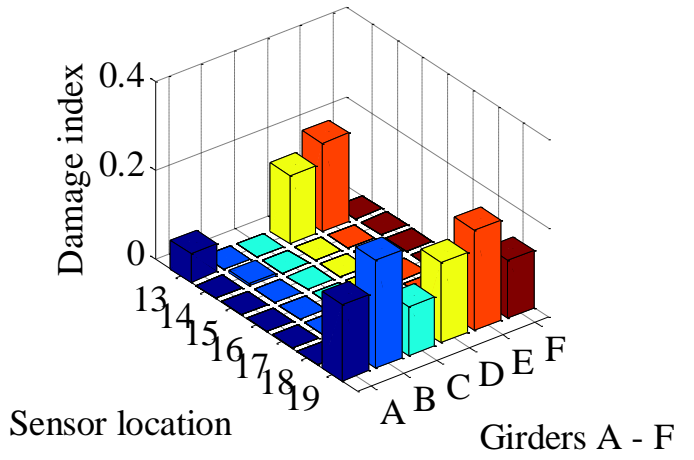

Figure 24 - Damage detection results for Bridge No.852 (a) with RDFRF, left span; (b) with RDFRF, right span; (c) with transmissibility, left span; (d) with transmissibility, right span 